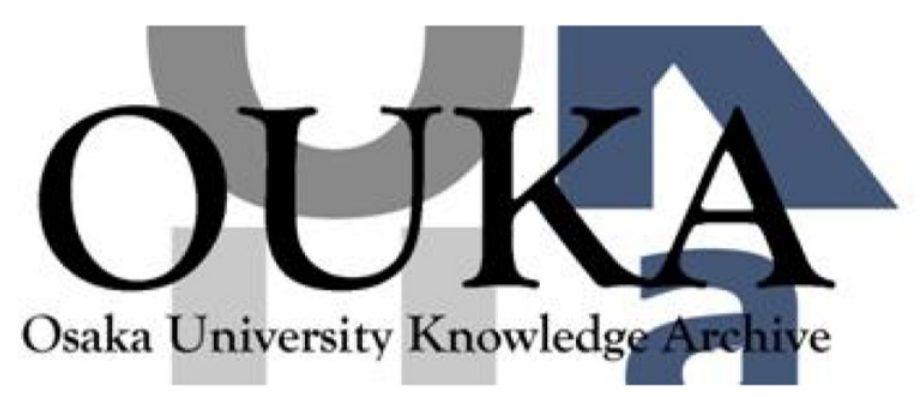

\begin{tabular}{|c|c|}
\hline Title & $\begin{array}{l}\text { Construction of nonlinear lattice with } \\
\text { potential symmetry for smooth propagation of } \\
\text { discrete breather }\end{array}$ \\
\hline Author (s) & Doi, Yusuke; Yoshimura, Kazuyuki \\
\hline Citation & Nonlinearity. 33(10) p. 5142-p. 5175 \\
\hline Issue Date & $2020-08-26$ \\
\hline oaire:version & AM \\
\hline URL & https://hdl. handle. net/11094/84549 \\
\hline rights & $\begin{array}{l}\text { ब2020 The Japan Society of Applied Physics. } \\
\text { This Accepted Manuscript is available for reuse } \\
\text { under a Creative Commons Attribution- } \\
\text { NonCommercial-NoDerivatives } 4.0 \text { International } \\
\text { License after the } 12 \text { month embargo period } \\
\text { provided that all the terms of the license are } \\
\text { adhered to. }\end{array}$ \\
\hline Note & \\
\hline
\end{tabular}

Osaka University Knowledge Archive : OUKA

https://ir. Library. osaka-u. ac. jp/

Osaka University 


\title{
Construction of Nonlinear Lattice with Potential Symmetry for Smooth Propagation of Discrete Breather
}

\author{
Yusuke Doi \\ Department of Adaptive Machine Systems, Graduate School of Engineering, Osaka \\ University \\ 2-1 Yamadaoka, Suita, Osaka 565-0871, Japan \\ E-mail: doi@ams.eng.osaka-u.ac.jp
}

\section{Kazuyuki Yoshimura}

Faculty of Engineering, Tottori University

4-101 Koyama-Minami, Tottori 680-8552, Japan

E-mail: kazuyuki@tottori-u.ac.jp

PACS numbers:

\begin{abstract}
We construct a nonlinear lattice that has a particular symmetry in its potential function consisting of long-range pairwise interactions. The symmetry enhances smooth propagation of discrete breathers, and it is defined by an invariance of the potential function with respect to a map acting on the complex normal mode coordinates. Condition of the symmetry is given by a set of algebraic equations with respect to coefficients of the pairwise interactions. We prove that the set of algebraic equations has a unique solution, and moreover we solve it explicitly. We present an explicit Hamiltonian for the symmetric lattice, which has coefficients given by the solution. We demonstrate that the present symmetric lattice is useful for numerically computing traveling discrete breathers in various lattices. We propose an algorithm using it.
\end{abstract}




\section{Introduction}

Wave propagation is one of the fundamental phenomena in physics. It has been of crucial importance to understand the nature of wave propagation in spatially discrete media. For example, phonons are plane waves in crystals, and their characteristics dominate various material properties such as thermal transport. In addition to the discreteness, a variety of discrete media are inherently nonlinear. Therefore, wave propagation in nonlinear discrete media has been of increasing importance.

Discrete breathers (DBs) are space-localized modes that ubiquitously emerge in a variety of nonlinear discrete media. The concept of DB was introduced by Takeno and Sievers [1], and it has been of great interest [2,3]. Two types of DBs are known to be possible, i.e., stationary and traveling DBs. Long-lived traveling DBs have been found numerically in various nonlinear lattice models $[4,5,6,7]$ : they propagate along the lattices without noticeable decay for a long time. Such traveling DBs are of considerable interest from the viewpoint of energy transport, and their properties have been investigated $[8,9,10,11]$.

Discreteness effects manifest in propagation property of traveling DB. The lattice discreteness in general tends to reduce the mobility of DB: for instance, an approximate traveling DB produced by perturbing a stationary DB loses its velocity during its propagation, and it is eventually trapped at a certain lattice site [7]. On the other hand, it is possible to precisely compute a traveling DB solution without velocity loss by combining the Newton method with numerical integration of the equations of motion. A remarkable feature is that it does not propagate with a constant velocity but with periodically varying velocity, i.e., the non-smooth propagation $[9,12]$. The period of this velocity variation is just a time needed for propagating one lattice space. Both the velocity loss of an approximate traveling $\mathrm{DB}$ and the velocity variation of a precise traveling DB vanish in the continuum limit, where the DB is very weakly localized $[10,13]$. This fact indicates that the two features are just manifestations of the lattice discreteness effects.

A fundamental issue is to clarify the origin of such discreteness effects, in other words, an essential property of the lattice potential that causes the discreteness effects. Addressing this issue, we pointed out the relevance of a particular symmetry of the lattice potential [10]. Recently, we have proposed a nonlinear lattice having this symmetry, which has a potential function consisting of pairwise long-range interactions. We have numerically demonstrated that this lattice allows constant-velocity traveling DBs and moreover exhibits a high mobility of approximate traveling DBs, i.e., the lattice is free from the discreteness effect [12]. In contrast, it is possible to break the symmetry by adding a perturbation to the lattice potential. As the perturbation increases, the velocity variation of precise traveling DB becomes larger (cf. Sec. 5) and the velocity loss of approximate traveling DB also increases [12]. These results indicate that breaking the symmetry is the origin of the discreteness effects in propagation of DBs.

It also should be emphasized that the ballistic thermal transport has been 
numerically observed in lattices with the potential symmetry $[16,17,18]$. In addition, when the symmetry is broken by adding a perturbation of the potential, the lattice exhibits a transition from the ballistic to a non-ballistic (but still anomalous) thermal transport as the lattice size increases [17]. The threshold lattice size $N_{c}$ depends on the magnitude of the perturbation: $N_{c}$ decreases as the perturbation is increased. These observations indicate that the thermal resistance appears and becomes stronger as the symmetry breaks gradually. Thus, it is expected that a study of the thermal transport by gradually breaking the symmetry in the present lattice will lead to a better understanding of the mechanism of thermal resistance. The present symmetric lattice may be of much significance also from the point of view of thermal transport.

In our previous paper [12], we stated that there exists such a symmetric lattice without a proof. In the present paper, we give a proof of its existence. Moreover, we give an explicit Hamiltonian for the symmetric lattice. Analytical expressions for the coefficients appearing in the symmetric lattice's potential were not given, but they were only numerically obtained in [12]. Here, we obtain the coefficients explicitly.

The symmetric lattice is useful for numerically computing traveling DB solutions. Precise computation of traveling DB usually employs the Newton method, which needs a good approximate solution as an initial guess. This approximation is obtained by perturbing a stationary DB, but this method does not always work successfully because the domain of convergence of the Newton method is very small for ordinary nonlinear lattices such as the Fermi-Pasta-Ulam (FPU) lattice and the approximation is not precise enough. A useful feature of the symmetric lattice is that it is possible to find a precise enough approximation by perturbation. Given a lattice model to compute a traveling DB such as the FPU one, our idea for the algorithm is to introduce a lattice that has a potential function parameterized between the symmetric lattice and the given one, compute a precise traveling DB for the symmetric lattice case, and then continue it to the given lattice case by gradually changing the parameter value. This idea has already been proposed in $[14,15]$. However, only the four-particle symmetric lattice was constructed at that point, and the $N$-particle one has been lacking. The present lattice is an extension of the four-particle symmetric lattice to an arbitrary degrees of freedom. We demonstrate that our algorithm using the present lattice successfully works for computing traveling DBs in the FPU lattice.

The rest of paper is organized as follows. In section 2, the definition of the symmetric lattice is given. In section 3, we give a pairwise interaction symmetric lattice, as well as the main results on the existence and uniqueness of the proposed model. The explicit expression of the proposed model is also given. In section 4, the numerical method for finding traveling DBs using the proposed model is presented. In section 5, we discuss physical effects of breaking the symmetry. In section 6 , we give a preliminary discussion of the proof. In section 7 and 8, several lemmas are proved to prepare the proof of main result. The proof of main result is given in section 9 . 


\section{Definition of symmetric lattice}

Let us consider a nonlinear lattice described by the Hamiltonian

$$
H=\frac{1}{2} \sum_{n=1}^{N} p_{n}^{2}+\Phi\left(q_{1}, q_{2}, \ldots, q_{N}\right),
$$

where $p_{n} \in \mathbb{R}$ and $q_{n} \in \mathbb{R}$ represent the linear momentum and the position of $n$th particle, respectively, $N$ is the number of particles of the system, and $\Phi\left(q_{1}, q_{2}, \ldots, q_{N}\right)$ : $\mathbb{R}^{N} \rightarrow \mathbb{R}$ is a $C^{2}$ function of $\left(q_{1}, q_{2}, \ldots, q_{N}\right)$. We assume the case of even $N$ and the periodic boundary conditions $p_{N+1}=p_{1}, p_{0}=p_{N}, q_{N+1}=q_{1}$ and $q_{0}=q_{N}$.

Consider the variable transformation defined by

$$
q_{n}=\frac{(-1)^{n}}{\sqrt{N}} \sum_{m=-N / 2+1}^{N / 2} U_{m} \exp \left[-i \frac{2 \pi n}{N} m\right], \quad n=1,2, \cdots, N,
$$

where $U_{m} \in \mathbb{C}, m=-N / 2+1,-N / 2+2, \ldots, N / 2$ are called the complex normal mode coordinates. Note that the $N / 2$ th mode represents the uniform displacement of the lattice. Substituting Eq. (2), Hamiltonian (1) can be written in terms of $U_{m}$ and reads

$$
H=\frac{1}{2} \sum_{m=-N_{h}}^{N_{h}+1} \dot{U}_{m} \dot{U}_{-m}+\Phi\left(\mathbf{U}, U_{N / 2}\right),
$$

where $N_{h}=N / 2-1$ and $\mathbf{U}=\left(U_{-N_{h}}, U_{-N_{h}+1}, \ldots, U_{N_{h}}\right)$. The potential $\Phi\left(\mathbf{U}, U_{N / 2}\right)$ can be decomposed as

$$
\Phi\left(\mathbf{U}, U_{N / 2}\right)=\Phi(\mathbf{U}, 0)+\mathcal{G}\left(\mathbf{U}, U_{N / 2}\right),
$$

where $\mathcal{G}\left(\mathbf{U}, U_{N / 2}\right) \equiv \Phi\left(\mathbf{U}, U_{N / 2}\right)-\Phi(\mathbf{U}, 0)$.

Consider the map $\mathcal{T}_{\lambda}: \mathbb{C}^{N-1} \rightarrow \mathbb{C}^{N-1}$ defined by

$$
\mathcal{T}_{\lambda}: U_{m} \mapsto U_{m} \exp (-i m \lambda), \quad m=-N_{h}, \ldots, N_{h},
$$

where $\lambda$ is a real parameter. This map $\mathcal{T}_{\lambda}$ forms a one-parameter transformation group. When $\lambda=2 \pi / N$, given an arbitrary displacement pattern $\mathbf{q}=\left(q_{1}, q_{2}, \cdots, q_{N}\right)$ satisfying $U_{N / 2}=\sum_{n=1}^{N} q_{n} / \sqrt{N}=0$, the map $\mathcal{T}_{\lambda}$ represents successive operations of shifting $\mathbf{q}$ by one lattice spacing and reversing the sign of the resulting displacement. Therefore, $\mathcal{T}_{\lambda}$ with an arbitrary $\lambda$ may be regarded as a continuous extension of this one-lattice-space shifting and sign-inverting transformation.

The $U_{N / 2}$-independent part of potential function $\Phi(\mathbf{U}, 0)$ in Eq. (4) can be divided into two parts: $\Phi_{\mathrm{s}}(\mathbf{U})$ and $\Phi_{\mathrm{a}}(\mathbf{U})$. The former part is invariant with respect to the map $\mathcal{T}_{\lambda}$ for any $\lambda \in \mathbb{R}$ and any $\mathbf{U} \in \mathbb{C}^{N-1}$, i.e., $\Phi_{\mathrm{s}}\left(\mathcal{T}_{\lambda} \mathbf{U}\right)=\Phi_{\mathrm{s}}(\mathbf{U})$, while $\Phi_{\mathrm{a}}(\mathbf{U})=\Phi(\mathbf{U}, 0)-\Phi_{\mathrm{s}}(\mathbf{U})$ is the rest part of $\Phi(\mathbf{U}, 0)$ and not invariant with respect to $\mathcal{T}_{\lambda}$ for any $\lambda$. We call the former part $\Phi_{\mathrm{s}}(\mathbf{U})$ the symmetric part. This decomposition in Eq.(3) provides

$$
H=\frac{1}{2} \sum_{m=-N_{h}}^{N_{h}+1} \dot{U}_{m} \dot{U}_{-m}+\Phi_{\mathrm{s}}(\mathbf{U})+\Psi\left(\mathbf{U}, U_{N / 2}\right),
$$


where $\Psi=\Phi_{\mathrm{a}}(\mathbf{U})+\mathcal{G}\left(\mathbf{U}, U_{N / 2}\right)$ is the asymmetric part of the whole potential $\Phi\left(\mathbf{U}, U_{N / 2}\right)$. Let $\mathcal{I}=\left\{(\mathbf{q}, \mathbf{p}) \in \mathbb{R}^{2 N} \mid \sum_{n=1}^{N} q_{n}=\sum_{n=1}^{N} p_{n}=0\right\}$. This is the subspace which is specified by $U_{N / 2}=\dot{U}_{N / 2}=0$. We give the following definition.

Definition 1. The lattice (3) or (6) is said to be a symmetric lattice if $\mathcal{I}$ is an invariant subspace and $\Psi(\mathbf{U}, 0)=0$.

By this definition, the Hamiltonian of the reduced dynamical system on $\mathcal{I}$ of a symmetric lattice is given in the form

$$
H=\frac{1}{2} \sum_{m=-N_{h}}^{N_{h}} \dot{U}_{m} \dot{U}_{-m}+\Phi_{\mathrm{s}}(\mathbf{U})
$$

It has been reported that the following two propositions hold in the symmetric lattice[15].

Proposition 2. Suppose that the symmetric lattice (7) has a solution $U_{m}(t)=$ $u_{m}(t), m=-N_{h}, \ldots, N_{h}, U_{N / 2}(t)=0$. Then for any $\lambda \in \mathbb{R}, U_{m}(t)=$ $u_{m}(t) \exp (-i m \lambda), m=-N_{h}, \ldots, N_{h}, U_{N / 2}(t)=0$ is also a solution.

Proposition 3. The symmetric lattice (7) has an additional first integral given by

$$
I=\sum_{m=1}^{N / 2-1} m\left(\dot{U}_{m} U_{-m}-U_{m} \dot{U}_{-m}\right)
$$

\section{Model and main result}

It is possible to construct a symmetric lattice from any lattice system defined by Hamiltonian (3), because it is enough to eliminate its asymmetric terms. However, such a model is in general unphysical when it is transformed into the Hamiltonian in terms of $q_{n}$. For example, such lattice is not composed of pairwise interactions. Our purpose is to construct a symmetric lattice that has a potential consisting of pairwise interactions only.

In section 2, we considered the symmetric lattice defined for even $N$. For simplicity of the proof, we restrict the following discussion to the case that $N$ is a multiple of 4 , i.e, $N / 2$ is even. We have not given the proof for the case that $N / 2$ is odd. However, it may be possible to prove as the same manner. Let us consider the Hamiltonian which has pairwise interaction terms as follows:

$$
H=\sum_{n=1}^{N} \frac{1}{2} p_{n}^{2}+\sum_{n=1}^{N}\left[\frac{\mu_{0}}{2} q_{n}^{2}+\frac{\mu_{1}}{2}\left(q_{n+1}-q_{n}\right)^{2}\right]+\frac{1}{4} \sum_{n=1}^{N} \sum_{r=1}^{N / 2} b_{r}\left(q_{n+r}-q_{n}\right)^{4},
$$

where $b_{r} \in \mathbb{R}$ is a constant which represents the coupling strength between the $r$ th neighboring particles, $\mu_{0}$ and $\mu_{1}$ are the coefficients of harmonic on-site potential and harmonic intersite potential, respectively. Hamiltonian (9) reduces to the FPU-type lattice when $\mu_{0}=0$ and $b_{r}=0 \quad(r \geq 2)$. 
Substituting Eq. (2) into Eq. (9), we rewrite the Hamiltonian in terms of $U_{m}$ into the form

$$
H=\frac{1}{2} \sum_{m=-N_{h}}^{N_{h}+1} \dot{U}_{m} \dot{U}_{-m}+\Phi_{\mathrm{s}}(\mathbf{U})+\Phi_{\mathrm{a}}(\mathbf{U})+\frac{\mu_{0}}{2} U_{N / 2}^{2}
$$

It is easy to see that Hamiltonian (10) has the invariant subspace $\mathcal{I}$. Comparing Eq.(10) with Eq.(6), we see $\Psi\left(\mathbf{U}, U_{N / 2}\right)=\Phi_{\mathrm{a}}(\mathbf{U})+\left(\mu_{0} / 2\right) U_{N / 2}^{2}$. This asymmetric part reduces $\Psi(\mathbf{U}, 0)=\Phi_{\mathrm{a}}(\mathbf{U})$ on $\mathcal{I}$. In order to construct a symmetric lattice, it is enough to consider $\Phi_{\mathrm{a}}(\mathbf{U})$ as the asymmetric part. The symmetric part and asymmetric part in the lattice potential are given as follows:

$$
\begin{aligned}
\Phi_{\mathrm{S}}(\mathbf{U})= & \frac{1}{2} \sum_{m=-N_{h}}^{N_{h}}\left[\mu_{0}+4 \mu_{1} \cos ^{2}\left(\frac{\pi m}{N}\right)\right] U_{m} U_{-m} \\
+ & \frac{4}{N} \sum_{i, j, k, l=-N_{h}}^{N_{h}} \phi^{(i, j, k, l)}(\mathbf{b}) U_{i} U_{j} U_{k} U_{l} \Delta(i+j+k+l) \\
\Phi_{\mathrm{a}}(\mathbf{U})= & -\frac{4}{N} \sum_{i, j, k, l=-N_{h}}^{N_{h}} \psi^{(i, j, k, l)}(\mathbf{b}) U_{i} U_{j} U_{k} U_{l} \Delta(i+j+k+l+N) \\
& -\frac{4}{N} \sum_{i, j, k, l=-N_{h}}^{N_{h}} \psi^{(i, j, k, l)}(\mathbf{b}) U_{i} U_{j} U_{k} U_{l} \Delta(i+j+k+l-N)
\end{aligned}
$$

with

$$
\begin{aligned}
\phi^{(i, j, k, l)}(\mathbf{b}) & =\sum_{q=1}^{N / 2} b_{q} f_{q}^{(i, j, k, l)} \\
\psi^{(i, j, k, l)}(\mathbf{b}) & =-\sum_{q=1}^{N / 2}(-1)^{q} b_{q} f_{q}^{(i, j, k, l)},
\end{aligned}
$$

where $\mathbf{b}=\left[b_{1}, b_{2}, \ldots, b_{N / 2}\right]^{T}$ and

$$
f_{q}^{(i, j, k, l)}= \begin{cases}c_{i q} c_{j q} c_{k q} c_{l q} & \text { for odd } q \\ s_{i q} s_{j q} s_{k q} s_{l q} & \text { for even } q,\end{cases}
$$

where $c_{\alpha}=\cos (\alpha \pi / N)$ and $s_{\alpha}=\sin (\alpha \pi / N)$. The function $\Delta(d)$ is defined by

$$
\Delta(d)= \begin{cases}1 & \text { if } d=0 \\ 0 & \text { otherwise }\end{cases}
$$

Let $\mathcal{S}_{ \pm}=\left\{(i, j, k, l) \in \mathbb{Z}^{4} \mid-N_{h} \leq i, j, k, l \leq N_{h}, i+j+k+l= \pm N\right\}$. The lattice (10) becomes symmetric if and only if the asymmetric part (12) vanishes. The condition $\Phi_{\mathrm{a}}(\mathbf{U})=0$ is equivalent to

$$
\psi^{(i, j, k, l)}(\mathbf{b})=0, \quad \forall(i, j, k, l) \in \mathcal{S}_{ \pm} .
$$

As for Eq. (17), we can readily obtain the following lemma. 
Lemma 4. Suppose that $\psi^{(i, j, k, l)}(\mathbf{b})=0$ holds for $(i, j, k, l) \in \mathbb{Z}^{4}$ and $\mathbf{b} \in \mathbb{R}^{N / 2}$, then we have $\psi^{(-i,-j,-k,-l)}(\mathbf{b})=0$.

Proof: For any $q \in \mathbb{Z}, s_{-(2 q-1) i}=-s_{(2 q-1) i}$ and $c_{-2 q i}=c_{2 q i}$ hold. This fact implies $f_{q}^{(-i,-j,-k,-l)}=f_{q}^{(i, j, k, l)}$. Therefore, we have $\psi^{(-i,-j,-k,-l)}(\mathbf{b})=\psi^{(i, j, k, l)}(\mathbf{b})$.

Since Eq.(17) is invariant under any exchange of indices, we can restrict our discussion to the subset $S_{0}=\left\{(i, j, k, l) \mid-N_{h} \leq i \leq j \leq k \leq l \leq N_{h}, i+j+k+l=\right.$ $\pm N\} \subset \mathcal{S}_{ \pm}$without loss of generality. Using this fact and Lemma 4 , we can further restrict our discussions to the set $S=\left\{(i, j, k, l) \mid-N_{h} \leq i \leq j \leq k \leq l \leq\right.$ $\left.N_{h}, i+j+k+l=N\right\} \subset S_{0}$. Finally, it is enough to discuss the equations in the set $S$ instead of Eq. (17) which is in the set $\mathcal{S}_{ \pm}$. Therefore, we consider the equations

$$
\psi^{(i, j, k, l)}(\mathbf{b})=0, \forall(i, j, k, l) \in S .
$$

Let $S_{1}=\{(0, n+1, N / 2-n, N / 2-1) \mid 1 \leq n \leq N / 4-1\} \subset S$ and $S_{2}=$ $\{(2-m, m, N / 2-1, N / 2-1) \mid m=1$ or $3 \leq m \leq N / 4+1\} \subset S$. The following lemma holds:

Lemma 5. Let $N \in \mathbb{N}$ be a multiple of 4. Equations $\psi^{(i, j, k, l)}(\mathbf{b})=0, \forall(i, j, k, l) \in S_{1} \cup S_{2}$ are $N / 2-1$ linearly independent equations and therefore they have a nontrivial solution $\mathbf{b} \neq 0$. Moreover, this nontrivial solution $\mathbf{b}$ also solves the other equations in $S$.

We briefly describe the procedure of our proof of Lemma 5 below:

(i) Showing that $N / 4-1$ equations $\psi^{(i, j, k, l)}(\mathbf{b})=0, \forall(i, j, k, l) \in S_{1}$ are linearly independent by showing the matrix rank of this set of equations is $N / 4-1$;

(ii) Showing that $N / 4$ equations $\psi^{(i, j, k, l)}(\mathbf{b})=0, \forall(i, j, k, l) \in S_{2}$ are linearly independent provided that (i) is satisfied, by showing the matrix rank of this set of equations is $N / 4$;

(iii) Showing that the nontrivial solution obtained by (i) and (ii) solves the other all equations $\psi^{(i, j, k, l)}(\mathbf{b})=0, \forall(i, j, k, l) \in S \backslash\left(S_{1} \cup S_{2}\right)$.

The proof of this lemma will be given in Secs. 7 and 8 .

The solution $\mathbf{b}$ of the set of equations in Lemma 5 can also be explicitly obtained as in Lemma 6. Its detailed derivation will be given in Appendix D.

Lemma 6. Let $N \in \mathbb{N}$ be a multiple of 4 and $b_{1}$ be a nonzero constant. The nontrivial solution of equations $\psi^{(i, j, k, l)}(\mathbf{b})=0, \forall(i, j, k, l) \in S_{1} \cup S_{2}$ is given by

$$
b_{r}= \begin{cases}\frac{b_{1} \sin ^{2} \frac{\pi}{N}}{\sin ^{2} \frac{r \pi}{N}} & (r=1,2, \cdots, N / 2-1), \\ \frac{b_{1}}{2} \sin ^{2} \frac{\pi}{N} & \left(r=\frac{N}{2}\right) .\end{cases}
$$

Combining Lemmas 5 and 6, we can obtain the following main theorem. The lattice model given in the following main theorem is called the pairwise interaction symmetric lattice (PISL)[12]. 
Theorem 7. Let $N \in \mathbb{N}$ be a multiple of 4 and $\mathbf{b}=\left(b_{1}, b_{2}, \cdots, b_{N / 2}\right)$ be a nontrivial solution of equations $\psi^{(i, j, k, l)}(\mathbf{b})=0, \forall(i, j, k, l) \in S_{1} \cup S_{2}$. Then, the lattice defined by the Hamiltonian (9) is a symmetric lattice, and the explicit expression of Hamiltonian (9) is given as follows:

$$
\begin{aligned}
H & =\frac{1}{2} \sum_{n=1}^{N} p_{n}^{2}+\frac{1}{2} \sum_{n=1}^{N}\left[\mu_{0} q_{n}^{2}+\mu_{1}\left(q_{n+1}-q_{n}\right)^{2}\right] \\
& +\frac{1}{4} \sum_{n=1}^{N} \sum_{r=1}^{N / 2-1} \frac{b_{1} \sin ^{2} \frac{\pi}{N}}{\sin ^{2} \frac{r \pi}{N}}\left(q_{n+r}-q_{n}\right)^{4}+\frac{1}{8} \sum_{n=1}^{N} b_{1} \sin ^{2}\left(\frac{\pi}{N}\right)\left(q_{n+N / 2}-q_{n}\right)^{4},
\end{aligned}
$$

where $b_{1}$ is an arbitrary nonzero constant.

\section{Calculation method for traveling DB}

It has been reported in our previous work[12] that one of the good features of the PISL is that DBs in the PISL have smooth mobility, that is, each traveling DB propagates with

a constant velocity. It has been also reported that the PISL has a rather large tolerance in the initial perturbation that generates a smoothly propagating traveling DB from a stationary DB. These results indicate the proposed model is useful for obtaining an initial guess for finding a traveling DB by iteration method. We propose the following procedure (i)-(vii) for computing a traveling DB solution, which utilizes the PISL.

(i) Consider the following lattice which has a parameter $C$ controlling the symmetry of lattice (PISL for $C=1$ and FPU- $\beta$ for $C=0$ ).

$$
\begin{aligned}
H= & \sum_{n=1}^{N}\left[\frac{1}{2} p_{n}^{2}+\frac{1}{2}\left(q_{n+1}-q_{n}\right)^{2}+\frac{b_{1}}{4}\left(q_{n+1}-q_{n}\right)^{4}\right] \\
& +\frac{C}{4} \sum_{n=1}^{N} \sum_{r=2}^{N / 2} b_{r}\left(q_{n+r}-q_{n}\right)^{4},
\end{aligned}
$$

where $b_{r}$ is given by Eq. (19). This lattice was named the translational asymmetry controlled lattice (TASCL) [15]. The equations of motion are given by

$$
\begin{aligned}
& \dot{q}_{n}=p_{n}, \\
& \dot{p}_{n}=q_{n+1}+q_{n-1}-2 q_{n}+b_{1}\left[\left(q_{n+1}-q_{n}\right)^{3}+\left(q_{n-1}-q_{n}\right)^{3}\right] \\
& \quad+C \sum_{r=2}^{N / 2} b_{r}\left[\left(q_{n+r}-q_{n}\right)^{3}+\left(q_{n-r}-q_{n}\right)^{3}\right],
\end{aligned}
$$

where $n=1,2, \cdots, N$. Denote $(\mathbf{q}, \mathbf{p})=\left(q_{1}, \cdots, q_{N}, p_{1}, \cdots, p_{N}\right)$. The temporal evolution of a solution with its initial condition $(\mathbf{q}(0), \mathbf{p}(0))$ is obtained by integrating Eqs. (22) and (23). This temporal evolution over the duration $\tau$ induces the map $\mathcal{F}_{C, \tau}: \mathbb{R}^{2 N} \rightarrow \mathbb{R}^{2 N}$ as follows:

$$
\mathcal{F}_{C, \tau}(\mathbf{q}(0), \mathbf{p}(0))=(\mathbf{q}(\tau), \mathbf{p}(\tau))
$$


(ii) Construct an approximate stationary DB with a prescribed angular frequency $\omega_{\mathrm{DB}}$ in the symmetric lattice $(C=1)$. Assume the approximate stationary DB solution in the form

$$
q_{n}(t)=a_{n} \cos \omega_{\mathrm{DB}} t, \quad n=1,2, \cdots, N
$$

where $a_{n}$ represents the spatial profile of stationary DB. Substituting Eq. (25) into Eqs. (22) and (23) and performing the rotating wave approximation, we obtain the algebraic equations for $a_{n}$ as follows:

$$
\begin{aligned}
& a_{n+1}+a_{n-1}-\left(2-\omega_{\mathrm{DB}}^{2}\right) a_{n}+\frac{3 b_{1}}{4}\left[\left(a_{n+1}-a_{n}\right)^{3}+\left(a_{n-1}-a_{n}\right)^{3}\right] \\
& +\frac{3}{4} \sum_{r=2}^{N / 2} b_{r}\left[\left(a_{n+r}-a_{n}\right)^{3}+\left(a_{n-r}-a_{n}\right)^{3}\right]=0, \quad n=1,2, \cdots, N .
\end{aligned}
$$

The particle amplitudes $a_{n}, n=1,2, \cdots N$, of the approximate stationary DB are obtained by numerically solving Eq.(26).

(iii) Construct a precise numerical solution of stationary DB with the angular frequency $\omega_{\mathrm{DB}}$ in the symmetric lattice $(C=1)$ under the constraint $U_{N / 2}=0$. This is performed by finding the periodic orbit in the phase space. Let $\left(\mathbf{q}^{(\mathrm{s})}(0), \mathbf{p}^{(\mathrm{s})}(0)\right)$ be the initial state of the stationary $\mathrm{DB}$ and $T=2 \pi / \omega_{\mathrm{DB}}$ be its internal oscillation period. The temporal evolution map $\mathcal{F}_{1, T}\left(\mathbf{q}^{(\mathrm{s})}(0), \mathbf{p}^{(\mathrm{s})}(0)\right)$ is defined by integration of Eqs. (22) and (23) with $C=1$ over the period $T$. The initial state has to satisfy the condition

$$
\mathcal{F}_{1, T}\left(\mathbf{q}^{(\mathrm{s})}(0), \mathbf{p}^{(\mathrm{s})}(0)\right)=\left(\mathbf{q}^{(\mathrm{s})}(0), \mathbf{p}^{(\mathrm{s})}(0)\right) .
$$

This is an equation for $\left(\mathbf{q}^{(\mathrm{s})}(0), \mathbf{p}^{(\mathrm{s})}(0)\right)$. Solve Eq. (27) by the Newton method with using $\left(a_{1}, \cdots, a_{N}, 0, \cdots, 0\right)$ as an initial guess for $\left(\mathbf{q}^{(\mathrm{s})}(0), \mathbf{p}^{(\mathrm{s})}(0)\right)$.

(iv) Construct an approximate traveling $\mathrm{DB}$ with velocity $v_{\mathrm{DB}}=r / s[\mathrm{site} /$ period] in the symmetric lattice $(C=1)$ under the constraint $U_{N / 2}=0$ by adding small perturbation to the stationary DB obtained in step (iii). The parameters $r$ and $s$ are integers. This means that the traveling DB propagates $r$ lattice spacings during $s$ internal oscillating periods $s T$, where $T=2 \pi / \omega_{\mathrm{DB}}$. It is natural to take the perturbation parallel to the direction $d \mathcal{T}_{\lambda}[\mathbf{U}] / d \lambda$, since the map $\mathcal{T}_{\lambda}$ represents a translational shift of DB along the lattice. Let $\delta U_{m}$ be each component of the perturbation vector, and we set

$$
\delta U_{m}=-i m U_{m} \cdot \delta l, \quad m=-N / 2+1, \cdots, N / 2-1
$$

from a simple calculation of $d / d \lambda\left(U_{m} \exp (-i m \lambda)\right)=-i m U_{m} \exp (-i m \lambda)$ and $\delta U_{N / 2}=0$ from $U_{N / 2}=0$. The parameter $\delta l$ determines the magnitude of perturbation. The perturbation $\delta \mathbf{p}=\left(\delta p_{1}, \delta p_{2}, \cdots, \delta p_{N}\right)$ in the physical space is given by

$$
\delta p_{n}=-\delta l \frac{(-1)^{n}}{\sqrt{N}} \sum_{m=-N / 2+1}^{N / 2} i m U_{m} \exp \left[-i \frac{2 \pi n}{N} m\right], \quad n=1,2, \cdots, N
$$


Fig. 1 shows the relation between the parameter $\delta l$ and the velocity $v_{\mathrm{DB}}$ of the traveling DB constructed by the perturbation (29). The detailed procedure for estimating the velocity $v_{\mathrm{DB}}$ of the approximate traveling $\mathrm{DB}$ is described in Appendix A. It is found that the velocity $v_{\mathrm{DB}}$ is proportional to $\delta l$ in a certain range. Therefore, the parameter $\delta l$ is adjusted so that the traveling DB has the prescribed velocity $v_{\mathrm{DB}}(\mathrm{cf}$. Appendix A). We obtain the initial state of the approximate traveling DB solution as $\overline{\mathbf{X}}^{(\mathrm{t})}(0)=\left(\mathbf{q}^{(\mathrm{s})}(0), \mathbf{p}^{(\mathrm{s})}(0)+\delta \mathbf{p}\right)$.

(v) Consider the TASCL with $C \in[0,1]$. For the prescribed values $T_{\mathrm{DB}}=2 \pi / \omega_{\mathrm{DB}}$ and $v_{\mathrm{DB}}=r / s$, define the map $\mathcal{M}_{C}: \mathbb{R}^{2 N} \rightarrow \mathbb{R}^{2 N}$ as follows:

$$
\mathcal{M}_{C}=(-1)^{r} \mathcal{S}_{r} \circ \mathcal{F}_{C, s T},
$$

where $\mathcal{S}_{r}: \mathbb{R}^{2 N} \rightarrow \mathbb{R}^{2 N}$ is the map that represents the cyclic permutation as follows:

$$
\mathcal{S}_{r}\left(q_{1}, \cdots, q_{N}, p_{1}, \cdots, p_{N}\right)=\left(q_{1-r}, \cdots, q_{N-r}, p_{1-r}, \cdots, p_{N-r}\right)
$$

Note that if the index $i-r(i=1,2, \cdots, N)$ of $q_{i-r}$ in RHS is not positive, it should be interpreted as $i-r+N$ since we consider periodic boundary conditions. Let $\left(\mathbf{q}^{(\mathrm{t})}(0), \mathbf{p}^{(\mathrm{t})}(0)\right)$ be the initial state of the traveling DB with $\omega_{\mathrm{DB}}$ and $v_{\mathrm{DB}}$. The initial state has to satisfy the condition

$$
\mathcal{M}_{C}\left(\mathbf{q}^{(\mathrm{t})}(0), \mathbf{p}^{(\mathrm{t})}(0)\right)=\left(\mathbf{q}^{(\mathrm{t})}(0), \mathbf{p}^{(\mathrm{t})}(0)\right)
$$

This is an equation for $\left(\mathbf{q}^{(\mathrm{t})}(0), \mathbf{p}^{(\mathrm{t})}(0)\right)$. It is possible to solve it by using the Newton method to find the solution precisely. Denote the solution of Eq. (32) with $\mathbf{X}_{C}^{(\mathrm{t})}(0)=\left(\mathbf{q}^{(\mathrm{t})}(0), \mathbf{p}^{(\mathrm{t})}(0)\right)$.

(vi) Consider the symmetric lattice $(C=1)$. Solve Eq. (32) by the Newton method with using $\overline{\mathbf{X}}^{(\mathrm{t})}(0)$ in step (iv) as an initial guess to obtain $\mathbf{X}_{1}^{(\mathrm{t})}(0)$.

(vii) Continue the solution $\mathbf{X}_{1}^{(\mathrm{t})}(0)$ in step (vi) to the solution $\mathbf{X}_{0}^{(\mathrm{t})}(0)$ for the FPU- $\beta$ lattice $(C=0)$. This continuation is performed by repeatedly solving Eq. (32) with gradually reducing the parameter $C$ until $C=0$. Let $\Delta C>0$ be a small constant and $\mathbf{X}_{C}^{(\mathrm{t})}(0)$ be the solution of Eq. (32) for $C$. Equation (32) for $C-\Delta C$ can be solved by using $\mathbf{X}_{C}^{(\mathrm{t})}(0)$ as an initial guess for the Newton method.

In steps (iii), (vi), and (vii), we have to find a fixed point $\mathbf{z}=(\mathbf{q}(0), \mathbf{p}(0))$ of a map by solving the equation of the form

$$
\mathcal{F}[\mathbf{z}]=\mathbf{z},
$$

where $\mathcal{F}: \mathbb{R}^{2 N} \rightarrow \mathbb{R}^{2 N}$ is a continuously differentiable map. A fixed point $\mathbf{z}$ can be found by using the Newton method described below. Let $\mathbf{z}_{0}$ be a point that is close to the fixed point $\mathbf{z}$ of the map $\mathcal{F}$. Let $\boldsymbol{\Delta}=\mathbf{z}-\mathbf{z}_{0}$ be the deviation. Substituting $\mathbf{z}=\mathbf{z}_{0}+\boldsymbol{\Delta}$ into Eq. (33) and performing the Taylor expansion with respect to $\boldsymbol{\Delta}$, we obtain

$$
\mathcal{F}\left[\mathbf{z}_{0}\right]+D \mathcal{F} \cdot \boldsymbol{\Delta}-\left(\mathbf{z}_{0}+\boldsymbol{\Delta}\right) \simeq \mathbf{0}
$$


where $D \mathcal{F}$ is the Jacobian matrix of $\mathcal{F}$ evaluated at $\mathbf{z}_{0}$. From (34), we obtain

$$
\Delta=-(D \mathcal{F}-I)^{-1}\left(\mathcal{F}\left[\mathbf{z}_{0}\right]-\mathbf{z}_{0}\right) .
$$

Equation (35) gives the improved approximation $\mathbf{z}_{0}^{\prime}=\mathbf{z}_{0}+\boldsymbol{\Delta}$. We can obtained an accurate numerical solution of Eq. (33) by repeating this calculation until $|\boldsymbol{\Delta}|$ becomes sufficiently small.

In the case of temporal evolution map $\mathcal{F}_{C, T}$, its Jacobian matrix $D \mathcal{F}_{C, T}$ can be evaluated from the variational equation of Eqs. (22) and (23). which is given by

$$
\begin{aligned}
\dot{\xi}_{n} & =\eta_{n}, \\
\dot{\eta}_{n} & =\xi_{n+1}+\xi_{n-1}-2 \xi_{n} \\
& +3 b_{1}\left[\left(q_{n+1}-q_{n}\right)^{2}\left(\xi_{n+1}-\xi_{n}\right)+\left(q_{n-1}-q_{n}\right)^{2}\left(\xi_{n-1}-\xi_{n}\right)\right] \\
& +3 C \sum_{r=2}^{N / 2} b_{r}\left[\left(q_{n+r}-q_{n}\right)^{2}\left(\xi_{n+r}-\xi_{n}\right)+\left(q_{n-r}-q_{n}\right)^{2}\left(\xi_{n-r}-\xi_{n}\right)\right]
\end{aligned}
$$

where $n=1,2, \cdots, N, \xi_{n}$ and $\eta_{n}$ are variations in $q_{n}$ and $p_{n}$, respectively. Integration of Eq. (37) over the period $T$ induces the temporal evolution map $\mathcal{G}_{T}: \mathbb{R}^{2 N} \rightarrow \mathbb{R}^{2 N}$ given by

$$
\mathcal{G}_{T}[(\xi(0), \eta(0))]=(\xi(T), \eta(T)),
$$

The Jacobian matrix $D \mathcal{F}_{C, T}$ is given by

$$
D \mathcal{F}_{C, T}=\left(\mathcal{G}_{T}\left[\boldsymbol{\Delta}_{1}\right]^{t}, \mathcal{G}_{T}\left[\boldsymbol{\Delta}_{2}\right]^{t}, \cdots, \mathcal{G}_{T}\left[\boldsymbol{\Delta}_{2 N}\right]^{t}\right),
$$

where the superscript $t$ stands for the transposition and $\boldsymbol{\Delta}_{n}$ is $2 N$ vector in which only $n$th component is one and the other components are zero.

In the case of map $\mathcal{M}_{C}$, its Jacobian matrix $D \mathcal{M}_{C}$, which is needed in steps (vi) and (vii), is given by

$$
D \mathcal{M}_{C}=(-1)^{r} S_{r} \circ\left(\mathcal{G}_{s T}\left[\boldsymbol{\Delta}_{1}\right]^{t}, \mathcal{G}_{s T}\left[\boldsymbol{\Delta}_{2}\right]^{t}, \cdots, \mathcal{G}_{s T}\left[\boldsymbol{\Delta}_{2 N}\right]^{t}\right) .
$$

where $S_{r}$ is the matrix that represents the map (31).

It should be noted that Eq. (27) in step (iii) is degenerate because of the translational invariance of equation of motion due to the conservation of total angular momentum and the arbitrariness of spatial symmetry of profile of stationary DB due to the extra conserved quantity of the symmetric lattices (see Eq. (8)). Therefore, we perform the Newton method under the constraint of $\sum_{n=1}^{N} q_{n}=0$ and keeping the spatial symmetry of profile of stationary DB, i.e., the even or odd mode. Moreover, Eq. (27) is degenerate because of the arbitrariness of the initial point along the periodic orbit. In order to remove this degeneracy, we consider the constraint of $\mathbf{p}^{(\mathrm{s})}(0)=\mathbf{0}[2]$.

As the same manner, Eq.(32) in steps (vi) and (vii) is degenerate because of the translational invariance of equation of motion and the arbitrariness of the initial point along the trajectory of the traveling DB. In order to remove this degeneracy, we perform the calculation under the constraints of $\sum_{n=1}^{N} q_{n}=\sum_{n=1}^{N} p_{n}=0$, and $q_{l}>0$ and $p_{l}=0$, where $l$ is the index of a particle that has the maximum amplitude. 
Examples of the numerical solutions obtained by the above-mentioned procedure are presented in Fig. 2. The internal period of $\mathrm{DB}$ is $T_{\mathrm{DB}}=2$ and the velocity $v_{\mathrm{DB}}=1 / 10$. Fig. 2 shows particle energy profiles of DBs with different values of $C$. The particle energy is defined by

$$
\begin{aligned}
e_{n}= & \frac{1}{2} p_{n}^{2}+\frac{1}{4}\left[\left(q_{n}-q_{n-1}\right)^{2}+\left(q_{n+1}-q_{n}\right)^{2}\right] \\
& +\frac{b_{1}}{8}\left[\left(q_{n}-q_{n-1}\right)^{4}+\left(q_{n+1}-q_{n}\right)^{4}\right] \\
& +\frac{C}{8} \sum_{r=2}^{N / 2} b_{r}\left[\left(q_{n}-q_{n-r}\right)^{4}+\left(q_{n+r}-q_{n}\right)^{4}\right] .
\end{aligned}
$$

In the symmetric case $(C=1)$, the traveling DB has no constant tail. By decreasing $C$, the spatially extended tail gradually appears. The trajectory of averaged center position of traveling DB with different $C$ is presented in Fig. 3. The center position of traveling DB is defined by

$$
x(t)=\sum_{n=1}^{N} n e_{n} .
$$

We perform the short-time average of $x(t)$ by

$$
X(t)=\frac{1}{2 \tau} \int_{t-\tau}^{t+\tau} x(t) d t
$$

in order to reduce fluctuations of $x(t)$ due to the internal vibration of traveling DB. Figure 3 shows the averaged center position $X(t)$. The DB travels with a constant velocity in the symmetric case $(C=1)$. The slope of the trajectory is $1 / 20$, which is equal to $v_{\mathrm{DB}} / T$. In the FPU- $\beta$ lattice case $(C=0)$, the velocity of DB periodically varies, but the averaged slope of the trajectory still coincides with $1 / 20$. These numerical results in Figs. 2 and 3 demonstrate that the proposed calculation method works well and successfully computes the traveling DB in the FPU- $\beta$ lattice.

In step (iv), we can obtain a good approximate traveling DB in the PISL which has a constant velocity. This is quite different from the case in the FPU- $\beta$ lattice because a traveling DB constructed from the perturbation gradually decreases its velocity. An advantage of the proposed method is that it is possible to obtain the traveling DB with a constant velocity easily.

In this section, we focus on the calculation method for the traveling DB in the FPU- $\beta$ lattice. The proposed calculation method may apply to compute traveling DBs by the continuation in the other nonlinear lattices such as the nonlinear Klein-Gordon lattices and FPU- $\alpha \beta$ lattice, provided that no bifurcation occurs during the continuation from the PISL. 


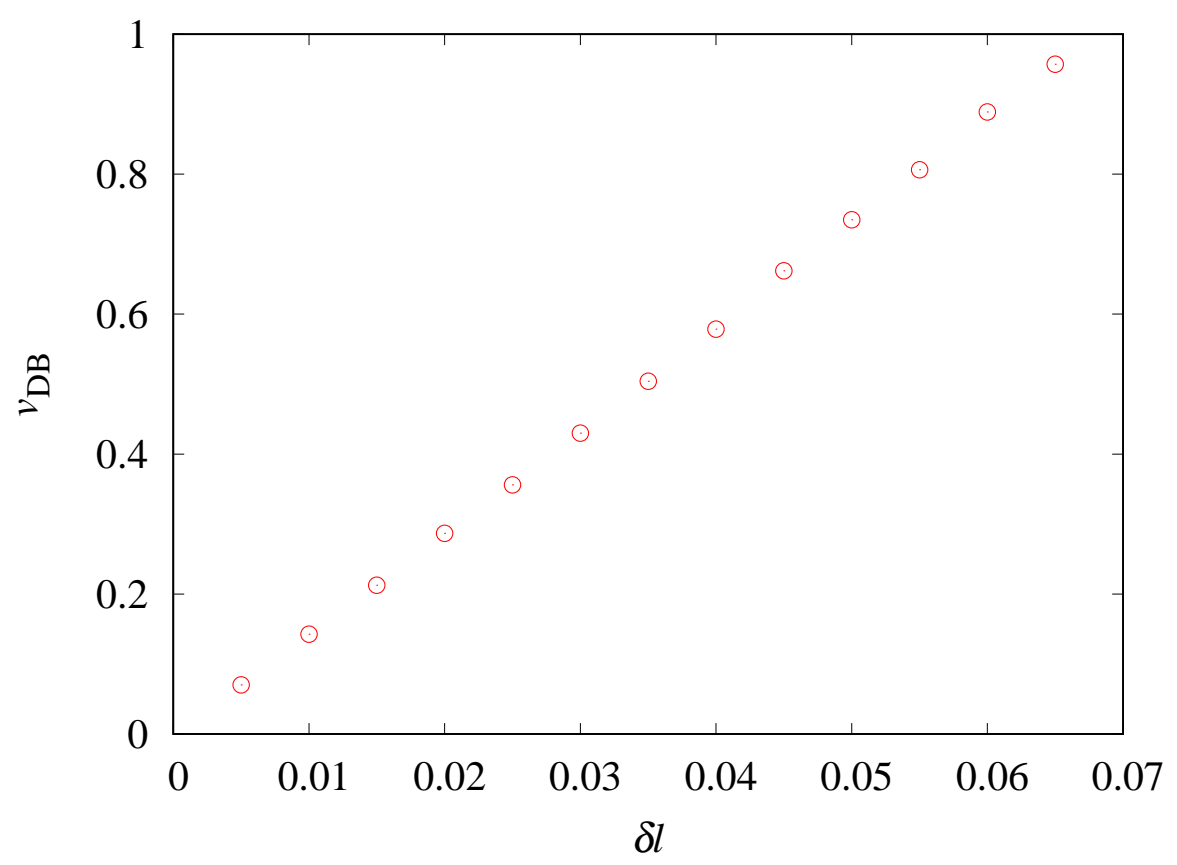

Figure 1. Relation between $\delta l$ and the velocity of approximate traveling DB $v_{\mathrm{DB}}$. The period of internal vibration of the stationary $\mathrm{DB}$ is $T_{\mathrm{DB}}=2$. 


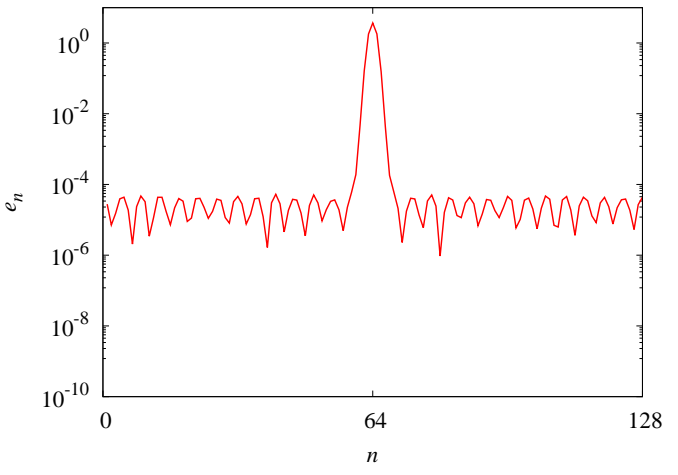

(a) $C=0.0$

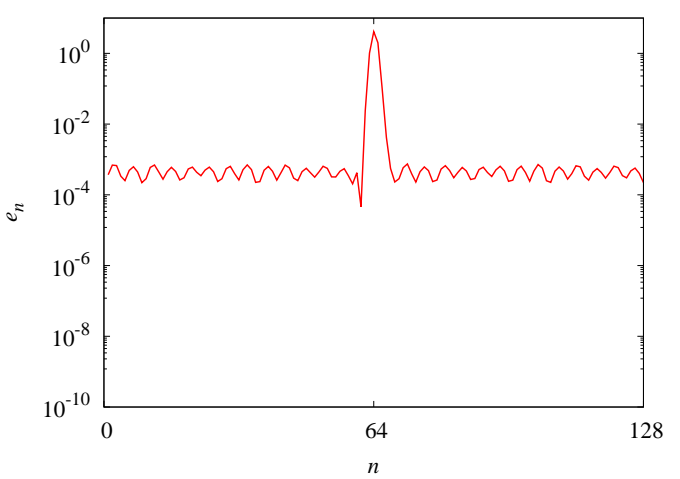

(c) $C=0.4$

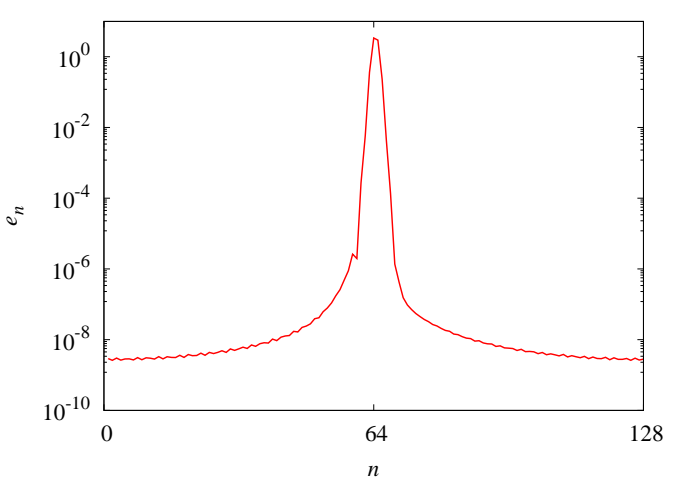

(e) $C=0.8$

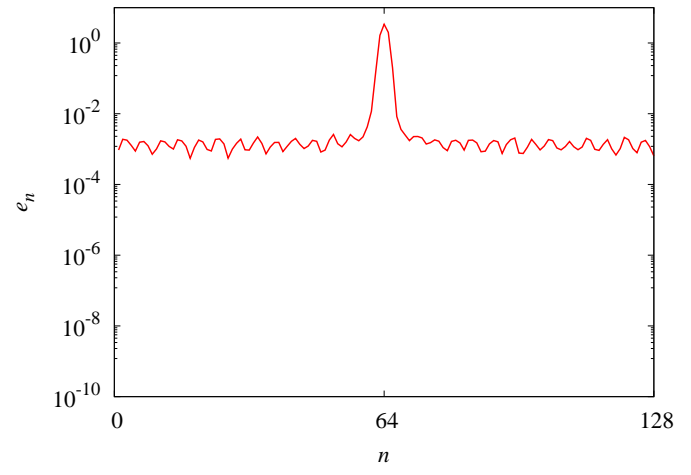

(b) $C=0.2$

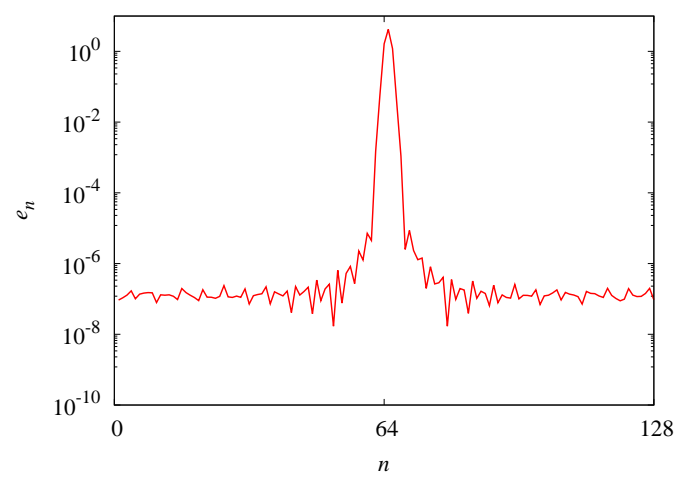

(d) $C=0.6$

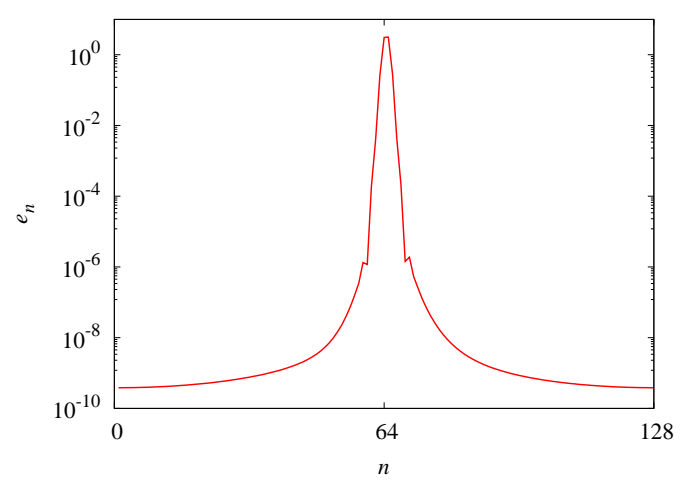

(f) $C=1.0$

Figure 2. Energy profile of DBs obtained by the iteration method, $T_{\mathrm{DB}}=2$ and $v_{\mathrm{DB}}=1 / 10$. 


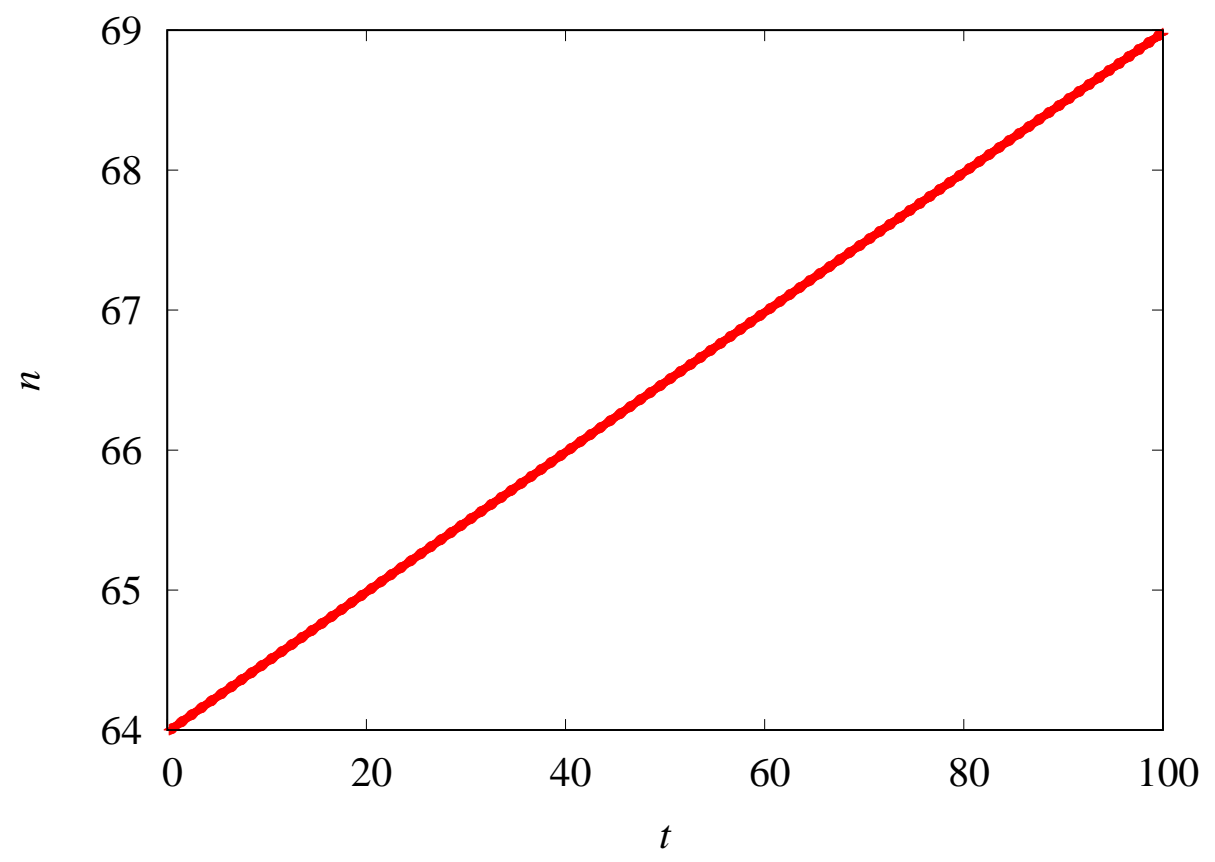

(a) $C=1$

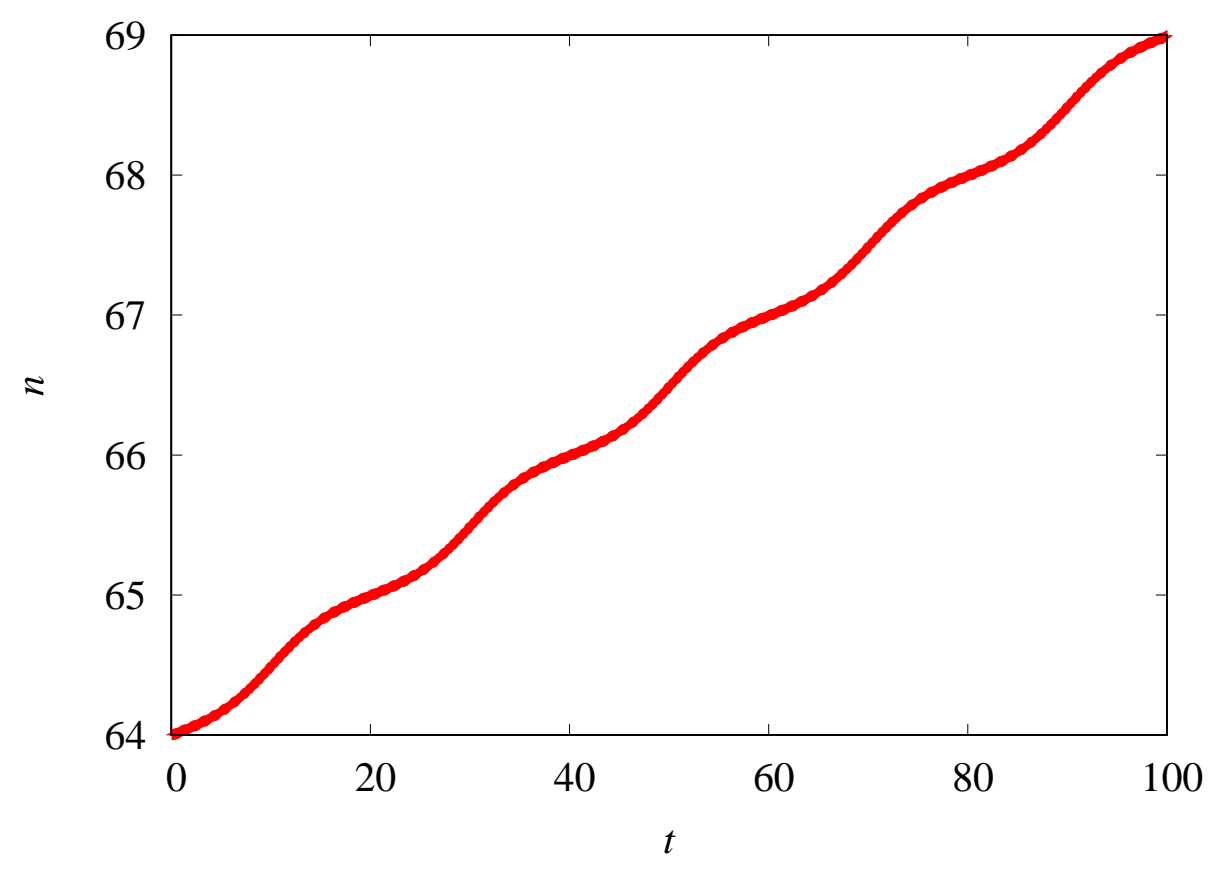

(b) $C=0$

Figure 3. Trajectory of traveling $\mathrm{DB}$ with $v_{\mathrm{DB}}=1 / 10$ and $T_{\mathrm{DB}}=2 \pi / \omega_{\mathrm{DB}}=2$ in (a)symmetric lattice $(C=1)$ and (b)FPU- $\beta$ lattice $(C=0)$ with $N=128$. 


\section{Truncated PISL and effects of breaking symmetry}

It is useful for investigating effects of breaking the symmetry to construct a truncated $P I S L$, in which only up to $M$-th nearest neighbor interactions are considered:

$$
\begin{aligned}
H & =\frac{1}{2} \sum_{n=1}^{N} p_{n}^{2}+\frac{1}{2} \sum_{n=1}^{N}\left[\mu_{0} q_{n}^{2}+\mu_{1}\left(q_{n+1}-q_{n}\right)^{2}\right] \\
& +\frac{1}{4} \sum_{n=1}^{N} \sum_{r=1}^{M} b_{r}\left(q_{n+r}-q_{n}\right)^{4} .
\end{aligned}
$$

The lattice (44) can be regarded as the PISL with the perturbation term $\Delta H$ as follows:

$$
\Delta H(M)=-\frac{1}{4} \sum_{n=1}^{N} \sum_{r=M+1}^{N / 2} b_{1}\left(q_{n+r}-q_{n}\right)^{4} .
$$

This perturbation breaks the symmetry of lattice. The parameter $M$ corresponds to the magnitude of perturbation. As the parameter $M$ becomes smaller, the magnitude of perturbation becomes larger.

When the perturbation (45) is introduced, the averaged center position $X(t)$ of a traveling $\mathrm{DB}$ deviates from the straight line $x_{\mathrm{s}}(t)=\left(v_{\mathrm{DB}} / T_{\mathrm{DB}}\right) t$ which corresponds to the case of a constant velocity. Figure 4 shows the deviation for different values of $M$. The magnitude of deviation becomes larger as $M$ decreases, i.e., the magnitude of perturbation becomes larger. It can be concluded that one of the symmetry breaking effects is the variation of DB's velocity. In addition to this effect, the symmetry breaking causes the velocity loss of approximate traveling DB (cf. Supplemental Material of [12]) and a degradation of the ballistic thermal transport observed in the symmetric case [17].

\section{Decomposition of Eq. (18)}

We have assumed that $N$ is a multiple of 4 . Under this assumption, equations (18) can be rewritten to

$$
c_{i} c_{j} c_{k} c_{l} b_{1}-s_{2 i} s_{2 j} s_{2 k} s_{2 l} b_{2} \ldots-s_{\frac{N}{2} i} s_{\frac{N}{2} j} s_{\frac{N}{2} k} s_{\frac{N}{2}} b_{N / 2}=0 .
$$

The function $\psi^{(i, j, k, l)}(\mathbf{b})$ can be decomposed into two parts as follows:

$$
\psi^{(i, j, k, l)}(\mathbf{b})=\psi_{\text {odd }}^{(i, j, k, l)}\left(\mathbf{b}_{\text {odd }}\right)-\psi_{\text {even }}^{(i, j, k, l)}\left(\mathbf{b}_{\text {even }}\right),
$$

where

$$
\begin{aligned}
& \psi_{\text {odd }}^{(i, j, k, l)}\left(\mathbf{b}_{\text {odd }}\right)=\sum_{s=1}^{N / 4} b_{2 s-1} c_{(2 s-1) i} c_{(2 s-1) j} c_{(2 s-1) k} c_{(2 s-1) l}, \\
& \psi_{\text {even }}^{(i, j, k, l)}\left(\mathbf{b}_{\text {even }}\right)=\sum_{s=1}^{N / 4} b_{2 s} s_{2 s i} s_{2 s j} s_{2 s k} s_{2 s l},
\end{aligned}
$$

and, $\mathbf{b}_{\text {odd }}=\left[b_{1}, b_{3}, \cdots, b_{N / 2-1}\right]^{T}$ and $\mathbf{b}_{\text {even }}=\left[b_{2}, b_{4}, \cdots, b_{N / 2}\right]^{T}$ are $N / 4$ vectors.

We will discuss the equations for $b_{2 s-1}$ and $b_{2 s}$ separately in the following sections. 


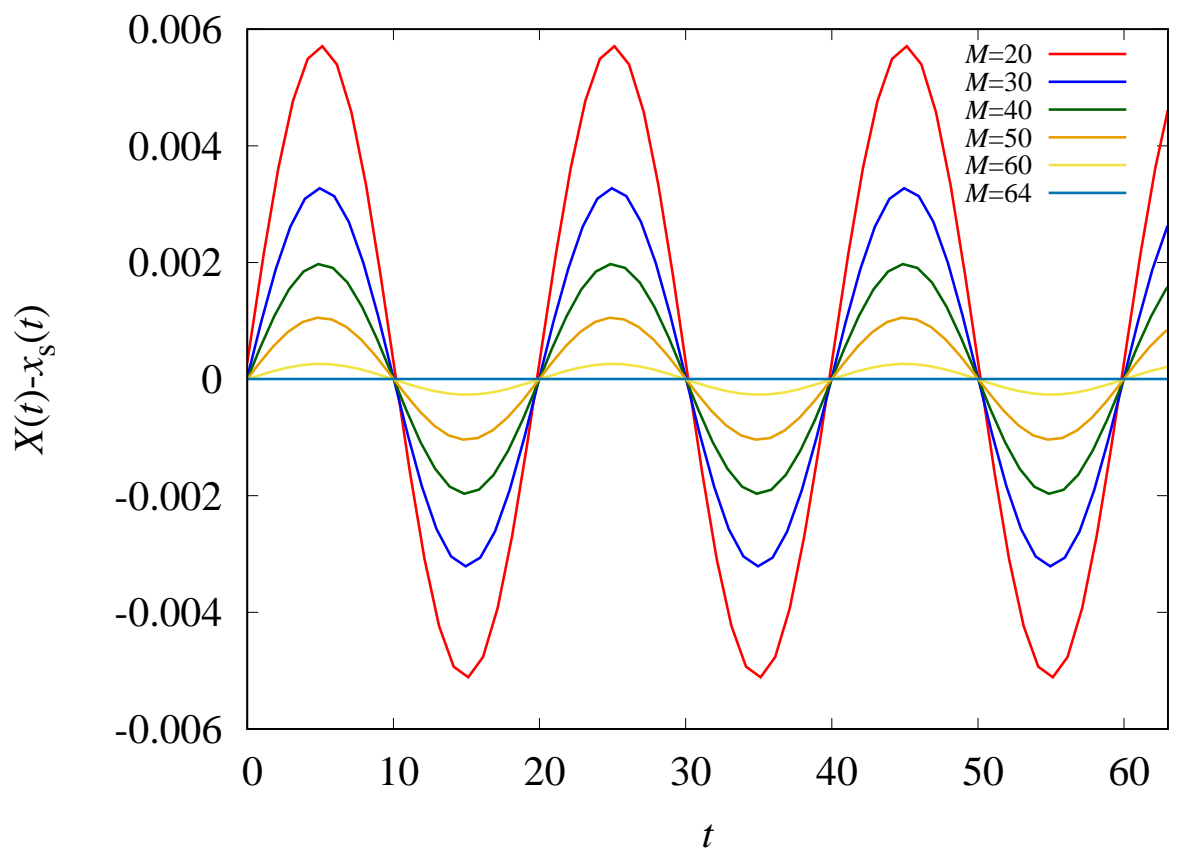

Figure 4. Deviation of the averaged position of traveling DB $X(t)$ from the straight trajectory $x_{\mathrm{s}}(t)=\left(v_{\mathrm{DB}} / T_{\mathrm{DB}}\right) t$ with $v_{\mathrm{DB}}=1 / 10$ and $T_{\mathrm{DB}}=2 \pi / \omega_{\mathrm{DB}}=2$ in the truncated PISL with $N=128$, and $M=20,30,40,50,60$. The case of full PISL $M=64$ is also presented.

\section{Equations for $b_{2 s-1}$}

The set $S$ can be divided into two subsets $T_{1}=\left\{(i=0, j, k, l) \mid-N_{h} \leq j \leq k \leq l \leq\right.$ $\left.N_{h}, j+k+l=N\right\} \subset S$ and $T_{2}=S \backslash T_{1}$. When $(i, j, k, l) \in T_{1}$, all terms corresponding to $s_{0}$ vanish. Therefore, $\psi^{(i, j, k, l)}(\mathbf{b})=0 \forall(i, j, k, l) \in T_{1}$ reduces to

$$
\psi_{\text {odd }}^{(i, j, k, l)}\left(\mathbf{b}_{\text {odd }}\right)=\sum_{s=1}^{N / 4} b_{2 s-1} c_{(2 s-1) i} c_{(2 s-1) j} c_{(2 s-1) k} c_{(2 s-1) l}=0 . \quad \forall(i, j, k, l) \in T_{1} .
$$

We consider the equations $\psi^{(i, j, k, l)}(\mathbf{b})=0 \forall(i, j, k, l) \in S_{1}=\{(0, n+1, N / 2-$ $n, N / 2-1) \mid 1 \leq n \leq N / 4-1\} \subset T_{1}$. Substituting $(i, j, k, l)=(0, n+1, N / 2-n, N / 2-1)$ into the LHS of (50), we obtain

$$
\begin{aligned}
\psi_{\text {odd }}^{(0, n+1, N / 2-n, N / 2-1)}\left(\mathbf{b}_{\text {odd }}\right) & =\sum_{s=1}^{N / 4} b_{2 s-1} c_{0} c_{(2 s-1)(n+1)} c_{(2 s-1)(N / 2-n)} c_{(2 s-1)(N / 2-1)} \\
& =\frac{1}{4} \sum_{s=1}^{N / 4} b_{2 s-1}\left[-1-c_{2(2 s-1)(n+1)}+c_{2(2 s-1)}+c_{2(2 s-1) n}\right]
\end{aligned}
$$


Therefore, equation (50) reduces to

$$
\begin{aligned}
& \frac{1}{4} \sum_{s=1}^{N / 4} b_{2 s-1}\left[-1-c_{2(2 s-1)(n+1)}+c_{2(2 s-1)}+c_{2(2 s-1) n}\right]=0 . \\
& \left(n=1,2, \ldots, \frac{N}{4}-1\right)
\end{aligned}
$$

Eq.(52) can be rewritten into the matrix form,

$$
\frac{1}{4} M_{1} A \mathbf{b}_{\text {odd }}=0,
$$

where $A$ is the $N / 4 \times N / 4$ matrix whose element is given by $A_{p q}=c_{2(p-1)(2 q-1)} . M_{1}$ is the $(N / 4-1) \times N / 4$ matrix defined by

$$
M_{1}=\left[\begin{array}{ccccccccc}
-1 & 2 & -1 & 0 & & & \cdots & 0 \\
-1 & 1 & 1 & -1 & 0 & & \cdots & 0 \\
-1 & 1 & 0 & 1 & -1 & 0 & & \cdots & 0 \\
\vdots & \vdots & & & & & \ddots & \\
& & & & & & & & \\
-1 & 1 & 0 & & & \cdots & 0 & 1 & -1 \\
-1 & 1 & 0 & & & \cdots & 0 & 0 & 1
\end{array}\right]
$$

Note that in the last row in $M_{1}$, we use the relation $c_{2(2 s-1) N / 4}=0$.

For the following discussion we introduce the $(N / 4-1) \times(N / 4-1)$ matrix $P_{1}$ defined by:

$$
P_{1}=\frac{1}{N / 4}\left[\begin{array}{ccccc}
1 & 1 & & \cdots & 1 \\
-(N / 4-2) & 2 & & \cdots & 2 \\
-(N / 4-3) & -(N / 4-3) & 3 & \cdots & 3 \\
\vdots & \vdots & & \ddots & \vdots \\
-1 & -1 & \cdots & -1 & N / 4-1
\end{array}\right]
$$

Lemma 8. $P_{1}$ is regular matrix.

Proof: Let a matrix $T_{1, p, q}$ be the elementary matrix which represents the elementary row operation of adding $q$ times of the first row to the $p$-th row. Applying $T_{1, m, N / 4-m} \quad(m=2,3, \cdots, N / 4-1)$ to $P_{1}$ from left, we obtain an upper triangular matrix as

$$
T_{1, N / 4-1,1} T_{1, N / 4-2,2} \cdots T_{1,2, N / 4-2} P_{1}=\frac{1}{N / 4}\left[\begin{array}{ccccc}
1 & 1 & 1 & \cdots & 1 \\
0 & N / 4 & N / 4 & \cdots & N / 4 \\
0 & 0 & N / 4 & \cdots & N / 4 \\
\vdots & \vdots & & \ddots & \vdots \\
0 & 0 & \cdots & 0 & N / 4
\end{array}\right] .
$$

The transformed upper triangular matrix is regular. Therefore $P_{1}$ is regular matrix. 
Applying $P_{1}$ to $M_{1}$, we obtain the $(N / 4-1) \times N / 4$ matrix

$$
P_{1} M_{1}=\left[\begin{array}{ccccc}
f_{N / 4}(1) & 1 & 0 & \cdots & 0 \\
f_{N / 4}(2) & 0 & 1 & \cdots & 0 \\
\vdots & \vdots & & \ddots & \vdots \\
f_{N / 4}(N / 4-1) & 0 & 0 & \cdots & 1
\end{array}\right]
$$

where $f_{m}(n)=-1+n / m$. It is clear from Eq.(57) that the rank of $P_{1} M$ is $N / 4-1$. Then, the following lemma holds:

Lemma 9. Rank of $M_{1}$ is $N / 4-1$.

The following proposition for the equations $\psi_{\text {odd }}^{(i, j, k)}\left(\mathbf{b}_{\text {odd }}\right)=0, \forall(i, j, k, l) \in S_{1}$ holds: Proposition 10. The equations $\psi_{\text {odd }}^{(i, j, l)}\left(\mathbf{b}_{\text {odd }}\right)=0, \forall(i, j, k, l) \in S_{1}$ are the $N / 4-1$ linearly independent equations for $b_{2 s-1}(s=1,2, \ldots, N / 4)$.

Proof: The rank of matrix $A$ is $N / 4$ (see Appendix B) and that of matrix $M_{1}$ is $N / 4-1$ from Lemma 9. Therefore, the rank of matrix $M_{1} A$ is $N / 4-1$. Therefore, the equations $\psi_{\text {odd }}^{(i, j, k, l)}\left(\mathbf{b}_{\text {odd }}\right)=0, \forall(i, j, k, l) \in S_{1}$ are $N / 4-1$ linearly independent equations for $b_{2 s-1}(s=1,2, \cdots, N / 4)$.

The equations $\psi_{\text {odd }}^{(i, j, k, l)}\left(\mathbf{b}_{\text {odd }}\right)=0, \forall(i, j, k, l) \in S_{1}$ are $N / 4-1$ linearly independent equations. On the other hand, the number of unknown variables $b_{2 s-1}$ is $N / 4$. Therefore, we can express the nontrivial solutions $b_{2 s-1}(s=2,3, \ldots, N / 4)$ in term of $b_{1}$ as follows:

$$
b_{2 s-1}=w_{2 s-1} b_{1},
$$

where $w_{2 s-1}$ is a real constant. Substituting (58) into (53) and then applying $P_{1}$ from the left side, we obtain the equations for $w_{2 s-1}$ :

$$
\sum_{s=1}^{N / 4}\left[f_{N / 4}(n)+c_{2 n(2 s-1)}\right] w_{2 s-1}=0
$$

for $n=1,2, \cdots, N / 4-1$. Note that we define $w_{1}=1$.

It can be easily confirmed that Eq.(59) also holds for the case of $n=0$ and $n=N / 4$. We next check that Eq.(59) also holds for a wider range of $n$. Define a new variable $n^{\prime}=N / 2-n$. Substituting $n=N / 2-n^{\prime}$ into Eq.(59), we obtain

$$
\sum_{s=1}^{N / 4}\left[f_{N / 4}\left(n^{\prime}\right)+c_{2 n^{\prime}(2 s-1)}\right] w_{2 s-1}=0
$$

for $n^{\prime}=N / 4+1, N / 4+2, \ldots, N / 2-1$. This indicates that Eq.(59) also holds for $n=N / 4+1, N / 4+2, \ldots, N / 2-1$. Finally, it is shown that Eq.(59) holds for the extended range $n=0,1, \ldots \ldots, N / 2-1$.

Next, we consider the equations $\psi_{\text {odd }}^{(i, j, k)}\left(\mathbf{b}_{\text {odd }}\right)=0, \forall(i, j, k, l) \in T_{1}$.

Proposition 11. The nontrivial solution $b_{2 s-1}=w_{2 s-1} b_{1}$ of the equations $\psi_{\text {odd }}^{(i, j, k)}\left(\mathbf{b}_{\text {odd }}\right)=0, \forall(i, j, k, l) \in S_{1}$ also solves the other equations in $T_{1}$. 
Proof: The set $T_{1}$ can be expressed by $(i, j, k, l)=(0, a+b, N / 2-b, N / 2-a)$, where $a$ and $b$ are integer which are in the ranges of $2 \leq a \leq N / 6$ and $a \leq b \leq(N-2 a) / 4$. Substituting solution (58) and using $\sum_{s=1}^{N / 4} c_{2 n(2 s-1)} w_{2 s-1}=-\sum_{s=1}^{N / 4} f_{N / 4}(n) w_{2 s-1}$, the left hand side of equations $\psi_{\text {odd }}^{(i, j, k, l)}\left(\mathbf{b}_{\text {odd }}\right)=0, \forall(i, j, k, l) \in T_{1}$ can be expressed as

$$
\begin{aligned}
& \psi_{\text {odd }}^{(0, a+b, N / 2-b, N / 2-a)}\left(w_{2 s-1} b_{1}\right) \\
& =b_{1} \sum_{s=1}^{N / 4} w_{2 s-1}\left[-1-c_{2(2 s-1)(a+b)}+c_{2(2 s-1) b}+c_{2(2 s-1) a}\right] \\
& =b_{1} \sum_{s=1}^{N / 4} w_{2 s-1}\left[-1+f_{N / 4}(a+b)-f_{N / 4}(b)-f_{N / 4}(a)\right] \\
& =0
\end{aligned}
$$

Therefore, the nontrivial solution $b_{2 s-1}=w_{2 s-1} b_{1}$ of the equations $\psi_{\text {odd }}^{(i, j, k, l)}\left(\mathbf{b}_{\text {odd }}\right)=$ $0, \forall(i, j, k, l) \in S_{1}$ also solves the other equations in $T_{1}$.

\section{Equations for $b_{2 s}$}

We consider $\psi^{(i, j, k, l)}(\mathbf{b})=0, \forall(i, j, k, l) \in S_{2}=\{(2-m, m, N / 2-1, N / 2-1) \mid m=$ 1 or $3 \leq m \leq N / 4+1\}$ under the condition that $b_{2 s-1}=w_{2 s-1} b_{1}$ is the nontrivial solution of $\psi_{\text {odd }}^{(i, j, k, l)}\left(\mathbf{b}_{\text {odd }}\right)=0, \forall(i, j, k, l) \in S_{1}$. Substituting Eq.(58) into $\psi^{(i, j, k, l)}(\mathbf{b})=$ $0, \quad \forall(i, j, k, l) \in S_{2}$, we obtain

$$
\psi_{\text {even }}^{(i, j, k, l)}\left(\mathbf{b}_{\text {even }}\right)=\mathcal{R}^{(i, j, k, l)}\left(w_{1}, w_{3}, \cdots, w_{N / 2-1}\right), \quad \forall(i, j, k, l) \in S_{2},
$$

where $\mathbf{b}_{\text {even }}=\left[b_{2}, b_{4}, \cdots, b_{N / 2}\right]^{T}$ is the $N / 4$-dimensional vector, and LHS and RHS of Eq.(62) are given as

$$
\begin{aligned}
\psi_{\text {even }}^{(i, j, k, l)}\left(\mathbf{b}_{\text {even }}\right) & =\sum_{s=1}^{N / 4} b_{2 s} s_{2 s i} s_{2 s j} s_{2 s k} s_{2 s l}, \\
\mathcal{R}^{(i, j, k, l)}\left(w_{1}, w_{3}, \cdots, w_{N / 2-1}\right) & =\psi_{\text {odd }}^{(i, j, k, l)}\left(\left\{b_{1} w_{2 s-1}\right\}_{s}\right) \\
& =\sum_{s=1}^{N / 4} b_{1} w_{2 s-1} c_{(2 s-1) i} c_{(2 s-1) j} c_{(2 s-1) k} c_{(2 s-1) l},
\end{aligned}
$$

respectively.

Substituting $i=2-m, j=m, k=N / 2-1, l=N / 2-1$, Eq. (63) can be rewritten as follows:

$$
\begin{aligned}
& \psi_{\text {even }}^{(2-m, N / 2-1, N / 2-1)}\left(\mathbf{b}_{\text {even }}\right) \\
& =\sum_{s=1}^{N / 4} b_{2 s} s_{2 s(2-m)} s_{2 s m} s_{2 s(N / 2-1)} s_{2 s(N / 2-1)} \\
& =\frac{1}{8} \sum_{s=1}^{N / 4}\left[1-2 c_{4 s}+c_{8 s}-c_{4 s(m-2)}+2 c_{4 s(m-1)}-c_{4 s m}\right] b_{2 s} .
\end{aligned}
$$


On the other hand, Eq.(64) becomes,

$$
\begin{aligned}
& \mathcal{R}^{(2-m, m, N / 2-1, N / 2-1)} \\
& =\sum_{s=1}^{N / 4} b_{1} w_{2 s-1} c_{(2 s-1)(2-m)} c_{(2 s-1) m} c_{(2 s-1)(N / 2-1)} c_{(2 s-1)(N / 2-1)} \\
& =\frac{b_{1}}{8} \sum_{s=1}^{N / 4} w_{2 s-1}\left[-1+2 c_{2(2 s-1)}-c_{4(2 s-1)}-c_{(2 s-1)(2 m-4)}+2 c_{(2 s-1)(2 m-2)}-c_{2 m(2 s-1)}\right] \\
& \quad\left(m=1, \text { or } 3 \leq m \leq \frac{N}{4}+1\right)
\end{aligned}
$$

Note that the sign of index of the fourth term in Eq.(66) can change depending on the value of $m$. In the case that $m=1$, the index becomes negative. The relation (59) is only valid for nonnegative index of $c_{n}$. We use the relation $c_{-n}=c_{n}$ to avoid the negative index. Substituting (59) into (66), we obtain

$$
\mathcal{R}^{(1,1, N / 2-1, N / 2-1)}=\frac{b_{1}}{N} \sum_{s=1}^{N / 4} \omega_{2 s-1} \equiv W b_{1} .
$$

When $m \geq 3$, substituting (59) into (66), we obtain

$$
\begin{aligned}
& \mathcal{R}^{(2-m, m, N / 2-1, N / 2-1)} \\
= & \frac{b_{1}}{8} \sum_{s=1}^{N / 4} w_{2 s-1}\left[-1-2 f_{N / 4}(1)+f_{N / 4}(2)+f_{N / 4}(m-2)-2 f_{N / 4}(m-1)+f_{N / 4}(m)\right] \\
= & 0 .
\end{aligned}
$$

Therefore, Eq.(62) is rewritten as

$$
\left\{\begin{array}{l}
\frac{1}{8} \sum_{s=1}^{N / 4}\left[3-4 c_{4 s}+c_{8 s}\right] b_{2 s}=W b_{1} \quad(m=1) \\
\frac{1}{8} \sum_{s=1}^{N / 4}\left[1-2 c_{4 s}+c_{8 s}-c_{4 s(m-2)}+2 c_{4 s(m-1)}-c_{4 s m}\right] b_{2 s}=0 . \quad\left(m=3,4, \ldots, \frac{N}{4}+1\right)
\end{array}\right.
$$

The matrix form of Eq. (69) is written as follows:

$$
\frac{1}{8} \tilde{M}_{2} \tilde{D} \mathbf{b}_{\text {even }}=\mathbf{g} \text {, }
$$

where $\tilde{M}_{2}$ is the $N / 4 \times(N / 4+1)$ matrix

$$
\tilde{M}_{2}=\left[\begin{array}{ccccccccc}
3 & -4 & 1 & 0 & & & & \cdots & 0 \\
1 & -3 & 3 & -1 & 0 & & & \cdots & 0 \\
1 & -2 & 0 & 2 & -1 & 0 & & \cdots & 0 \\
1 & -2 & 1 & -1 & 2 & -1 & 0 & \cdots & 0 \\
\vdots & \vdots & & & & & & \ddots & \\
& & & & & & & & \\
1 & -2 & 1 & 0 & \cdots & -1 & 2 & -1 & 0 \\
1 & -2 & 1 & 0 & \cdots & 0 & -1 & 2 & -1 \\
1 & -2 & 1 & 0 & & \cdots & 0 & -2 & 2
\end{array}\right],
$$


Note that in the last row in $\tilde{M}_{2}$, we use the relation $c_{4 s(N / 4+1)}=c_{4 s(N / 4-1)}$. $\tilde{D}$ is the $(N / 4+1) \times N / 4$ matrix that element is given by $D_{p q}=c_{4(p-1) q}$, and $\mathbf{g}$ is the $N / 4$ dimensional vector given by

$$
\mathbf{g}=\left[W b_{1}, 0, \cdots, 0\right]^{T} .
$$

Since $\tilde{M}_{2}$ and $\tilde{D}$ are not square matrix, we convert these matrices to square ones in order to proceed the proof.

Let $\tilde{\mathbf{x}}=\left[\tilde{x}_{1}, \tilde{x}_{2}, \cdots, \tilde{x}_{N / 4+1}\right]^{T}$ be the $(N / 4+1)$ vector defined as follows:

$$
\tilde{\mathbf{x}}=\tilde{D} \mathbf{b}_{\text {even }}
$$

or

$$
\tilde{x}_{i}=D_{i, 1} b_{2}+D_{i, 2} b_{4}+\cdots+D_{i, N / 4} b_{N / 2} . \quad(i=1,2, \cdots, N / 4+1) .
$$

Using the relation (73), Eq. (70) is rewritten as

$$
\frac{1}{8} \tilde{M}_{2} \tilde{\mathbf{x}}=\mathbf{g} .
$$

Let $\mathbf{m}_{i} \quad(i=1,2, \cdots, N / 4+1)$ be the column vectors of $\tilde{M}_{2}$. It can be easily checked that the last column vector $\mathbf{m}_{N / 4+1}=-\sum_{j=1}^{N / 4} \mathbf{m}_{j}$. Using this relation, Eq. (75) can be transformed into

$$
\frac{1}{8} M_{2} \mathbf{x}=\mathbf{g}
$$

where $M_{2}$ is the $N / 4 \times N / 4$ matrix

$$
M_{2}=\left[\begin{array}{ccccccccc}
3 & -4 & 1 & 0 & & & & \cdots & 0 \\
1 & -3 & 3 & -1 & 0 & & & \cdots & 0 \\
1 & -2 & 0 & 2 & -1 & 0 & & \cdots & 0 \\
1 & -2 & 1 & -1 & 2 & -1 & 0 & \cdots & 0 \\
\vdots & \vdots & & & & & & \ddots & \\
& & & & & & & & \\
1 & -2 & 1 & 0 & \cdots & 0 & -1 & 2 & -1 \\
1 & -2 & 1 & 0 & \cdots & 0 & 0 & -1 & 2 \\
1 & -2 & 1 & 0 & & \cdots & 0 & 0 & -2
\end{array}\right]
$$

and $\mathbf{x}$ is the $\mathrm{N} / 4$ vector defined by

$$
\begin{aligned}
\mathbf{x} & =\left[x_{1}, x_{2}, \cdots, x_{N / 4}\right]^{T} \\
& =\left[\tilde{x}_{1}-\tilde{x}_{N / 4+1}, \tilde{x}_{2}-\tilde{x}_{N / 4+1}, \cdots, \tilde{x}_{N / 4}-\tilde{x}_{N / 4+1}\right]^{T} .
\end{aligned}
$$

Next, we discuss the matrix $\tilde{D}$. Let $\mathbf{d}_{p}=\left[D_{p, 1}, D_{p, 2}, \cdots, D_{p, N / 4}\right] \quad(p=$ $1,2, \cdots, N / 4+1)$ be the row vectors of $\tilde{D}$. This immediately leads

$$
\begin{aligned}
& \mathbf{d}_{1}=[1,1, \cdots, 1] \\
& \mathbf{d}_{N / 4+1}=[-1,1, \cdots,-1,1]
\end{aligned}
$$

Moreover, it can be easily derived that

$$
\mathbf{d}_{1}+\mathbf{d}_{2}+\cdots+\mathbf{d}_{N / 4}=[1,0, \cdots, 1,0] \text {. }
$$


Therefore, we obtain the following relation

$$
\begin{aligned}
\mathbf{d}_{N / 4+1} & =\mathbf{d}_{1}-2\left(\mathbf{d}_{1}+\mathbf{d}_{2}+\cdots+\mathbf{d}_{N / 4}\right) \\
& =-\mathbf{d}_{1}-2\left(\mathbf{d}_{2}+\mathbf{d}_{3}+\cdots+\mathbf{d}_{N / 4}\right)
\end{aligned}
$$

or

$$
D_{N / 4+1, j}=-D_{1, j}-2\left(D_{2, j}+D_{3, j}+\cdots+D_{N / 4, j}\right) . \quad(j=1,2, \cdots, N / 4)
$$

Using the relation (74), the element of vector (78) can be rewritten as:

$$
\begin{aligned}
x_{i} & =\tilde{x}_{i}-\tilde{x}_{N / 4+1} \\
& =\left(D_{i, 1}-D_{N / 4+1,1}\right) b_{2}+\left(D_{i, 2}-D_{N / 4+1,2}\right) b_{4} \\
+ & \cdots+\left(D_{i, N / 4}-D_{N / 4+1, N / 4}\right) b_{N / 2} \\
& \quad(i=1,2, \cdots, N / 4)
\end{aligned}
$$

Substituting (83) into (84), we obtain

$$
\begin{aligned}
x_{1}= & 2\left(D_{1,1}+\cdots+D_{N / 4,1}\right) b_{2}+2\left(D_{1,2}+\cdots+D_{N / 4,2}\right) b_{4}+ \\
& \cdots+2\left(D_{1, N / 4}+\cdots D_{N / 4, N / 4}\right) b_{N / 2} \\
x_{i}= & \left(D_{1,1}+2 D_{2,1}+\cdots+3 D_{i, 1}+\cdots+2 D_{N / 4,1}\right) b_{2} \\
& +\left(D_{1,2}+2 D_{2,2}+\cdots+3 D_{i, 2}+\cdots+2 D_{N / 4,2}\right) b_{4} \\
& +\cdots+\left(D_{1, N / 4}+2 D_{2, N / 4}+\cdots+3 D_{i, N / 4}+\cdots+2 D_{N / 4, N / 4}\right) b_{N / 2} . \\
& \quad(i=2,3, \cdots, N / 4)
\end{aligned}
$$

The matrix form of Eq. (85) is

$$
\mathbf{x}=P_{4} D \mathbf{b}_{\text {even }}
$$

where $P_{4}$ is the $N / 4 \times N / 4$ matrix

$$
P_{4}=\left[\begin{array}{ccccccc}
2 & 2 & 2 & 2 & \ldots & 2 & 2 \\
1 & 3 & 2 & 2 & \ldots & 2 & 2 \\
1 & 2 & 3 & 2 & \ldots & 2 & 2 \\
1 & 2 & 2 & 3 & \ldots & 2 & 2 \\
\vdots & \vdots & \vdots & \vdots & \ddots & \vdots & \vdots \\
1 & 2 & 2 & 2 & \ldots & 3 & 2 \\
1 & 2 & 2 & 2 & \ldots & 2 & 3
\end{array}\right]
$$

and $D$ is the $N / 4 \times N / 4$ matrix constructed by removing the $(N / 4+1)$-th row from $\tilde{D}$.

Finally, we obtain the transformed equation of (70) by combining (76) and (86),

$$
\frac{1}{8} M_{2} P_{4} D \mathbf{b}_{\text {even }}=\mathbf{g} \text {. }
$$

As to the matrix $M_{2}$, the following lemma holds:

Lemma 12. $M_{2}$ is regular matrix. 
Nonlinear Lattice with Potential Symmetry for Smooth Propagation of DB

Proof: Consider the $N / 4 \times N / 4$ lower triangular matrix $P_{2}$ as follows

$$
P_{2}=\left[\begin{array}{cccccccc}
1 & 0 & 0 & 0 & \ldots & 0 & 0 & 0 \\
0 & 1 & 0 & 0 & \ldots & 0 & 0 & 0 \\
0 & 2 & 1 & 0 & \ldots & 0 & 0 & 0 \\
0 & 3 & 2 & 1 & \ldots & 0 & 0 & 0 \\
\vdots & \vdots & \vdots & \vdots & \ddots & 0 & 0 & 0 \\
0 & N / 4-3 & N / 4-4 & N / 4-5 & \ldots & 1 & 0 & 0 \\
0 & N / 4-2 & N / 4-3 & N / 4-4 & \ldots & 2 & 1 & 0 \\
0 & -2(N / 4-3) & -2(N / 4-4) & -2(N / 4-5) & \ldots & -2 & 0 & 1
\end{array}\right] .
$$

All diagonal components of the matrix $P_{2}$ are nonzero. Therefore $P_{2}$ is regular. Applying $P_{2}$ to $M_{2}$, we obtain

$$
P_{2} M_{2}=\left[\begin{array}{cccccccc}
3 & -4 & 1 & 0 & & & \cdots & 0 \\
a_{21} & a_{22} & a_{23} & -1 & 0 & & \cdots & 0 \\
a_{31} & a_{32} & a_{33} & 0 & -1 & 0 & \cdots & 0 \\
a_{41} & a_{42} & a_{33} & 0 & 0 & -1 & \cdots & 0 \\
\vdots & \vdots & & & & & \ddots & \\
a_{N / 4-3,1} & a_{N / 4-2,2} & a_{N / 4-2,3} & 0 & \cdots & 0 & 0 & -1 \\
a_{N / 4-1,1} & a_{N / 4-1,2} & a_{N / 4-1,3} & 0 & & \cdots & 0 & 0 \\
a_{N / 4,1} & a_{N / 4,2} & a_{N / 4,3} & 0 & & \cdots & 0 & 0
\end{array}\right],
$$

where

$$
\begin{aligned}
& a_{n, 1}= \begin{cases}n(n-1) / 2 & n=2,3, \ldots, N / 4-1 \\
-\left(N^{2}-20 N+80\right) / 16, & n=N / 4\end{cases} \\
& a_{n, 2}= \begin{cases}1-n^{2} & n=2,3, \ldots, N / 4-1 \\
\left(N^{2}-16 N+32\right) / 8, & n=N / 4\end{cases} \\
& a_{n, 3}= \begin{cases}n(n+1) / 2 & n=2,3, \ldots, N / 4-1 \\
-\left(N^{2}-12 N+16\right) / 16, & n=N / 4\end{cases}
\end{aligned}
$$

Finally, we consider the $N / 4 \times N / 4$ regular matrix $P_{3}$ as follows:

$$
P_{3}=\left[\begin{array}{cccccccc}
N / 16 & 0 & 0 & 0 & \cdots & 0 & 1-N / 8 & -N / 16 \\
0 & 1 & 0 & 0 & \cdots & 0 & 0 & 0 \\
0 & 0 & 1 & 0 & \cdots & 0 & 0 & 0 \\
\vdots & \vdots & & & \ddots & \vdots & \vdots \\
0 & 0 & 0 & 0 & \cdots & 1 & 0 & 0 \\
\frac{(N-4)^{2}}{16 N} & 0 & 0 & 0 & \cdots & 0 & 1+2 / N-N / 8 & 1 / N-N / 16 \\
\frac{(N-8)^{2}}{16 N} & 0 & 0 & 0 & \cdots & 0 & 1+8 / N-N / 8 & 4 / N-N / 16
\end{array}\right]
$$


Applying $P_{3}$ to $P_{2} M_{2}$, we obtain

$$
P_{3} P_{2} M_{2}=\left[\begin{array}{cccccccc}
1 & 0 & 0 & 0 & & & \cdots & 0 \\
a_{21} & a_{22} & a_{33} & -1 & 0 & & \cdots & 0 \\
a_{31} & a_{32} & a_{43} & 0 & -1 & 0 & \cdots & 0 \\
a_{41} & a_{42} & a_{53} & 0 & 0 & -1 & \cdots & 0 \\
\vdots & \vdots & & & & & \ddots & \\
a_{N / 4-2,1} & a_{N / 4-2,2} & a_{N / 4-2,3} & 0 & \cdots & 0 & 0 & -1 \\
0 & 1 & 0 & 0 & & \cdots & 0 & 0 \\
0 & 0 & 1 & 0 & & \cdots & 0 & 0
\end{array}\right] \text {. }
$$

We can eliminate the components $a_{i, j}, 2 \leq i \leq N / 4-2,1 \leq j \leq 3$ by elementary operations of adding the first, $N / 4-1$ and $N / 4$ rows. It is found that the rank of $P_{3} P_{2} M_{2}$ is $N / 4$. Since $P_{2}$ and $P_{3}$ is the regular matrix, the rank of $M_{2}$ is equal to $N / 4$. Therefore $M_{2}$ is regular.

As to equations $\psi_{\text {even }}^{(i, j, k, l)}\left(\mathbf{b}_{\text {even }}\right)=\sum_{s=1}^{N / 4} b_{1} w_{2 s-1} c_{(2 s-1) i} c_{(2 s-1) j} c_{(2 s-1) k} c_{(2 s-1) l}$, $\forall(i, j, k, l) \in S_{2}$, the following proposition holds:

Proposition 13. For a given solution $b_{2 n-1}=b_{1} w_{2 n-1}, n=1,2, \cdots N / 4$ for the equations $\psi_{\text {odd }}^{(i, j, k, l)}\left(\mathbf{b}_{\text {odd }}\right)=0, \forall(i, j, k, l) \in S_{1}$, equations $\psi_{\text {even }}^{(i, j, k, l)}\left(\mathbf{b}_{\text {even }}\right)=$ $\sum_{s=1}^{N / 4} b_{1} w_{2 s-1} c_{(2 s-1) i} c_{(2 s-1) j} c_{(2 s-1) k} c_{(2 s-1) l}, \forall(i, j, k, l) \in S_{2}$ are $N / 4$ linearly independent equations.

Proof: The equation (70) is equivalent to the transformed equation (88). $M_{2}$ is a $N / 4 \times N / 4$ regular matrix from Lemma 12 . Since $P_{4} D$ is the $N / 4 \times N / 4$ regular matrix(see Appendix C), $M_{2} P_{4} D$ is the $N / 4 \times N / 4$ regular matrix. Therefore, $\psi_{\text {even }}^{(i, j, k, l)}\left(\mathbf{b}_{\text {even }}\right)=\sum_{s=1}^{N / 4} b_{1} w_{2 s-1} c_{(2 s-1) i} c_{(2 s-1) j} c_{(2 s-1) k} c_{(2 s-1) l}, \forall(i, j, k, l) \in S_{2}$ are $N / 4$ linearly independent equations.

The solution of Eq. (88) can be written as $\mathbf{b}_{\text {even }}=8\left(P_{4} D\right)^{-1} M_{2}^{-1} \mathbf{g}$, which leads to $b_{2 s}=8\left[\left(P_{4} D\right)^{-1} M_{2}^{-1}\right]_{s, 1} W b_{1} \quad(s=1,2, \cdots, N / 4)$. Therefore, it can be also written in the form

$$
b_{2 s}=w_{2 s} b_{1}, \quad(s=1,2, \cdots, N / 4)
$$

where $w_{2 s}=8\left[\left(P_{4} D\right)^{(-1)} M_{2}^{-1}\right]_{s, 1} W$.

For converting Eq. (88) into a simple form, we introduce the $N / 4 \times N / 4$ regular matrices $Q_{2}, Q_{3}$ and $Q_{4}$ as follows:

$$
Q_{2}=\left[\begin{array}{ccccccc}
1 & 0 & 0 & \cdots & 0 & 0 & 0 \\
0 & 1 & 0 & \cdots & 0 & 0 & 0 \\
0 & 0 & 1 & \cdots & 0 & 0 & 0 \\
\vdots & \vdots & \vdots & \ddots & \vdots & \vdots & \vdots \\
0 & 0 & 0 & \cdots & 1 & 0 & 0 \\
0 & 2 & 2 & \cdots & 2 & 1 & 0 \\
0 & 0 & 0 & \cdots & 0 & 2 & 1
\end{array}\right]
$$


Nonlinear Lattice with Potential Symmetry for Smooth Propagation of DB

$$
\begin{aligned}
Q_{3} & =\left[\begin{array}{ccccccc}
1 & 0 & 0 & \cdots & 0 & 0 & 0 \\
0 & 1 & 0 & \cdots & 0 & 0 & 0 \\
0 & 0 & 1 & \cdots & 0 & 0 & 0 \\
\vdots & \vdots & \vdots & \ddots & \vdots & \vdots & \vdots \\
0 & 0 & 0 & \cdots & 1 & 0 & 0 \\
0 & 0 & 0 & \cdots & 0 & \frac{24}{N(N+2)} & -\frac{N-4}{4(N+2)} \\
0 & 0 & 0 & \cdots & 0 & \frac{48(N-4)}{N\left(N^{2}-4\right)} & -\frac{N^{2}-12 N+8}{2\left(N^{2}-4\right)}
\end{array}\right] \\
Q_{4} & =\left[\begin{array}{ccccccc}
1 & 0 & 0 & \cdots & 0 & 0 & 0 \\
0 & -1 & 0 & \cdots & 0 & a_{22} & a_{23} \\
0 & 0 & -1 & \cdots & 0 & a_{32} & a_{33} \\
\vdots & \vdots & \vdots & \ddots & \vdots & \vdots & \vdots \\
0 & 0 & 0 & \cdots & -1 & a_{N / 4-2,2} & a_{N / 4-2,3} \\
0 & 0 & 0 & \cdots & 0 & 1 & 0 \\
0 & 0 & 0 & \cdots & 0 & 0 & 1
\end{array}\right]
\end{aligned}
$$

Applying $Q_{4} Q_{3} Q_{2} P_{2}$ to (88), we obtain

$$
\frac{1}{8}\left[\begin{array}{cccccccc}
3 & -4 & 1 & 0 & 0 & 0 & \cdots & 0 \\
h_{N}(3) & 0 & 0 & 1 & 0 & 0 & \cdots & 0 \\
h_{N}(4) & 0 & 0 & 0 & 1 & 0 & \cdots & 0 \\
\vdots & \vdots & \vdots & \vdots & \vdots & & \ddots & \vdots \\
h_{N}(N / 4-1) & 0 & 0 & 0 & 0 & \cdots & 0 & 1 \\
h_{N}(1) & 1 & 0 & 0 & 0 & 0 & \cdots & 0 \\
h_{N}(2) & 0 & 1 & 0 & 0 & 0 & \cdots & 0
\end{array}\right] D \mathbf{b}_{\text {even }}=\mathbf{g} .
$$

where $h_{m}(n)=-1-\frac{24 n^{2}-12 n m}{m^{2}-4}$.

Substituting Eq. (96) into Eq. (100), the following relation holds:

$$
\frac{1}{8} \sum_{s=1}^{N / 4}\left[3-4 c_{4 s}+c_{8 s}\right] w_{2 s}=W
$$

and

$$
\frac{1}{8} \sum_{s=1}^{N / 4}\left[h_{N}(n)+c_{4 n s}\right] w_{2 s}=0 . \quad(n=1,2, \cdots, N / 4-1)
$$

It can be shown that Eq.(102) also holds for $n=0$ and $n=N / 2$. Define new variables $n^{\prime}=N / 2-n$. Substituting $n=N / 2-n^{\prime}$ into Eq.(102), we obtain

$$
\frac{1}{8} \sum_{s=1}^{N / 4}\left[h_{N}\left(n^{\prime}\right)+c_{4 n^{\prime} s}\right] w_{2 s}=0
$$

for $n^{\prime}=N / 4+1, N / 4+2, \cdots, N / 2-1$. Therefore, Eq.(102) holds for the extended range $n=0,1, \cdots, N / 2$. 
Substituting (102) for $n=1,2$ into (101), we obtain the relation

$$
\frac{3 N}{N^{2}-4} \sum_{s=1}^{N / 4} w_{2 s}=W
$$

We consider the equations $\psi_{\text {even }}^{(i, j, k)}\left(\mathbf{b}_{\text {even }}\right)=\sum_{s=1}^{N / 4} b_{1} w_{2 s-1} c_{(2 s-1) i} c_{(2 s-1) j} c_{(2 s-1) k} c_{(2 s-1) l}$, $\forall(i, j, k, l) \in T_{2}$.

Proposition 14. For the given solutions $b_{2 n-1}=b_{1} w_{2 n-1} \quad(n=1,2, \cdots N / 4)$ for the equations $\psi_{\text {odd }}^{(i, j, k)}\left(\mathbf{b}_{\text {odd }}\right)=0, \forall(i, j, k, l) \in S_{1}$, the nontrivial solution of equations $\psi_{\text {even }}^{(i, j, k, l)}\left(\mathbf{b}_{\text {even }}\right)=\sum_{s=1}^{N / 4} b_{1} w_{2 s-1} c_{(2 s-1) i} c_{(2 s-1) j} c_{(2 s-1) k} c_{(2 s-1) l}, \forall(i, j, k, l) \in S_{2}$ also solves the other equations in $T_{2}$.

Proof: The set $T_{2}$ can be expressed by $(i, j, k, l)=(a+b-c, c, N / 2-b, N / 2-a)$, where $a, b$ and $c$ are integers. Substituting $i=a+b-c, j=c, k=N / 2-b, l=N / 2-a$ into Eq.(63) which is LHS of Eq. (62), we obtain

$$
\begin{aligned}
& \psi_{\text {even }}^{(a+b-c, c, N / 2-b, N / 2-a)}\left(\mathbf{b}_{\text {even }}\right) \\
& =\sum_{s=1}^{N / 4} b_{2 s} s_{2(a+b-c) s} s_{2 c s} s_{(N-2 b) s} s_{(N-2 a) s} \\
& =\frac{1}{8} \sum_{s=1}^{N / 4}\left[1+c_{4(a+b) s}-c_{4(c-a-b) s}-c_{4 a s}-c_{4 b s}-c_{4 c s}+c_{4(c-a) s}+c_{4(c-b) s}\right] b_{2 s} .
\end{aligned}
$$

Since $i \leq j \leq k \leq l$, it is found that $(a+b) / 2 \leq c$ and $a \leq b$. Therefore, the relation $a \leq(a+b) / 2 \leq c$ holds. Then we have $c-a \geq 0$. Moreover, we have $a, b \geq 1$ since $N / 2-a \leq N / 2-1$ and $N / 2-b \leq N / 2-1$. Therefore, we have $1 \leq a \leq c$. In order to keep the index $\alpha$ of $c_{\alpha}$ positive or zero in the 3rd, 7th and last terms in Eq.(105), three cases of RHS of Eq. (105) should be considered.

(i) $a+b-c<0, c-a \geq 0$ and $c-b \geq 0$

$$
\begin{aligned}
L_{1}= & \frac{1}{8} \sum_{s=1}^{N / 4}\left[1+c_{4(a+b) s}-c_{4(c-a-b) s}-c_{4 a s}-c_{4 b s}-c_{4 c s}\right. \\
& \left.+c_{4(c-a) s}+c_{4(c-b) s}\right] b_{2 s}
\end{aligned}
$$

(ii) $a+b-c \geq 0, c-a \geq 0$ and $c-b \geq 0$

$$
\begin{aligned}
L_{2}= & \frac{1}{8} \sum_{s=1}^{N / 4}\left[1+c_{4(a+b) s}-c_{4(a+b-c) s}-c_{4 a s}-c_{4 b s}-c_{4 c s}\right. \\
& \left.+c_{4(c-a) s}+c_{4(c-b) s}\right] b_{2 s}
\end{aligned}
$$


(iii) $a+b-c \geq 0, c-a \geq 0$ and $c-b<0$

$$
\begin{aligned}
L_{3}= & \frac{1}{8} \sum_{s=1}^{N / 4}\left[1+c_{4(a+b) s}-c_{4(a+b-c) s}-c_{4 a s}-c_{4 b s}-c_{4 c s}\right. \\
& \left.+c_{4(c-a) s}+c_{4(b-c) s}\right] b_{2 s}
\end{aligned}
$$

Using (96) and (102), Eq.(106)-(108) are simplified as follows:

$$
\begin{aligned}
L_{1}= & \frac{b_{1}}{8} \sum_{s=1}^{N / 4}\left[1-h_{N}(a+b)+h_{N}(c-a-b)+h_{N}(a)+h_{N}(b)+h_{N}(c)\right. \\
& \left.-h_{N}(c-a)-h_{N}(c-b)\right] w_{2 s} \\
= & 0 . \\
L_{2}= & \frac{b_{1}}{8} \sum_{s=1}^{N / 4}\left[1-h_{N}(a+b)+h_{N}(a+b-c)+h_{N}(a)+h_{N}(b)+h_{N}(c)\right. \\
& \left.-h_{N}(c-a)-h_{N}(c-b)\right] w_{2 s} \\
= & \frac{3 N b_{1}}{N^{2}-4}(a+b-c) \sum_{s=1}^{N / 4} w_{2 s} . \\
L_{3}= & \frac{b_{1}}{8} \sum_{s=1}^{N / 4}\left[1-h_{N}(a+b)+h_{N}(a+b-c)+h_{N}(a)+h_{N}(b)+h_{N}(c)\right. \\
& \left.-h_{N}(c-a)-h_{N}(b-c)\right] w_{2 s} \\
= & \frac{3 N b_{1}}{N^{2}-4} a \sum_{s=1}^{N / 4} w_{2 s} .
\end{aligned}
$$

Substituting $i=a+b-c, j=c, k=N / 2-b, l=N / 2-a$ into Eq.(64) which is the RHS of Eq.(62), we obtain

$$
\begin{aligned}
\mathcal{R}^{(a+b-c, c, N / 2-b, N / 2-a)}= & \sum_{s=1}^{N / 4}\left[c_{(2 s-1)(a+b-c)} c_{(2 s-1) c} c_{(2 s-1)(N / 2-b)} c_{(2 s-1)(N / 2-a)}\right] b_{1} w_{2 s-1} \\
= & \frac{1}{8} \sum_{s=1}^{N / 4}\left[-1-c_{2(2 s-1)(a+b)}+c_{2(2 s-1) a}+c_{2(2 s-1) b}-c_{2(2 s-1) c}\right. \\
& \left.-c_{2(2 s-1)(a+b-c)}+c_{2(2 s-1)(a-c)}+c_{2(2 s-1)(b-c)}\right] b_{1} w_{2 s-1} .
\end{aligned}
$$

As same as the LHS, three cases should be considered in order to keep the index $\alpha$ of $c_{\alpha}$ positive or zero. In each case, the equation is simplified by using the relation (59). 
Nonlinear Lattice with Potential Symmetry for Smooth Propagation of DB

(i) $a+b-c<0, c-a \geq 0$ and $c-b \geq 0$

$$
\begin{aligned}
R_{1}= & \frac{b_{1}}{8} \sum_{s=1}^{N / 4}\left[-1-c_{2(2 s-1)(a+b)}+c_{2(2 s-1) a}+c_{2(2 s-1) b}\right. \\
& \left.-c_{2(2 s-1) c}-c_{2(2 s-1)(c-a-b)}+c_{2(2 s-1)(c-a)}+c_{2(2 s-1)(c-b)}\right] w_{2 s-1} \\
= & \frac{b_{1}}{8} \sum_{s=1}^{N / 4}\left[-1+f_{N / 4}(a+b)-f_{N / 4}(a)-f_{N / 4}(b)\right. \\
& \left.+f_{N / 4}(c)+f_{N / 4}(c-a-b)-f_{N / 4}(c-a)-f_{N / 4}(c-b)\right] w_{2 s-1} \\
= & 0
\end{aligned}
$$

(ii) $a+b-c \geq 0, c-a \geq 0$ and $c-b \geq 0$

$$
\begin{aligned}
R_{2}= & \frac{b_{1}}{8} \sum_{s=1}^{N / 4}\left[-1-c_{2(2 s-1)(a+b)}+c_{2(2 s-1) a}+c_{2(2 s-1) b}\right. \\
& \left.\quad-c_{2(2 s-1) c}-c_{2(2 s-1)(a+b-c)}+c_{2(2 s-1)(c-a)}+c_{2(2 s-1)(c-b)}\right] w_{2 s-1} . \\
= & \frac{b_{1}}{8} \sum_{s=1}^{N / 4}\left[-1+f_{N / 4}(a+b)-f_{N / 4}(a)-f_{N / 4}(b)\right. \\
& \left.+f_{N / 4}(c)+f_{N / 4}(a+b-c)-f_{N / 4}(c-a)-f_{N / 4}(c-b)\right] w_{2 s-1} \\
= & W b_{1}(a+b-c) .
\end{aligned}
$$

(iii) $a+b-c \geq 0, c-a \geq 0$ and $c-b<0$

$$
\begin{aligned}
R_{3}= & \frac{b_{1}}{8} \sum_{s=1}^{N / 4}\left[-1-c_{2(2 s-1)(a+b)}+c_{2(2 s-1) a}+c_{2(2 s-1) b}\right. \\
& \left.-c_{2(2 s-1) c}-c_{2(2 s-1)(a+b-c)}+c_{2(2 s-1)(c-a)}+c_{2(2 s-1)(b-c)}\right] w_{2 s-1} \\
= & \frac{b_{1}}{8} \sum_{s=1}^{N / 4}\left[-1+f_{N / 4}(a+b)-f_{N / 4}(a)-f_{N / 4}(b)\right. \\
& \left.+f_{N / 4}(c)+f_{N / 4}(a+b-c)-f_{N / 4}(c-a)-f_{N / 4}(b-c)\right] w_{2 s-1} \\
= & W b_{1} a .
\end{aligned}
$$

From the relation (104), it follows that $L_{i}-R_{i}=0 \quad(i=1,2,3)$. This indicates that the solution $\mathbf{b}_{\text {even }}$ of the equation $\psi_{\text {even }}^{(i, j, k, l)}\left(\mathbf{b}_{\text {even }}\right)=$ $\sum_{s=1}^{N / 4} b_{1} w_{2 s-1} c_{(2 s-1) i} c_{(2 s-1) j} c_{(2 s-1) k} c_{(2 s-1) l}, \forall(i, j, k, l) \in S_{2}$ also solves the other equations in $T_{2}$ when $b_{2 n-1}=b_{1} w_{2 n-1} \quad(n=1,2, \cdots, N / 4)$ is the solution of the equations $\psi_{\text {odd }}^{(i, j, k, l)}\left(\mathbf{b}_{\text {odd }}\right)=0, \forall(i, j, k, l) \in S_{1}$.

\section{Proof of Main Results}

Proof of Lemma 5. From Proposition 10 and Proposition 13, the equations $\psi^{(i, j, k, l)}(\mathbf{b})=0, \forall(i, j, k, l) \in S_{1} \cup S_{2}$ are $N-1$ linearly independent equations. Therefore, they have a nontrivial solution $\mathbf{b} \neq 0$ for given $b_{1}$. From Proposition 11 and Proposition 
14 , the nontrivial solution also solves the equations $\psi^{(i, j, k, l)}(\mathbf{b})=0, \forall(i, j, k, l) \in T_{1} \cup T_{2}=$ $S$. From Lemma 4, the solution also solves the equations for $S_{0}$.

Proof of Theorem 7. From Lemma 5, a trivial solution b for $\psi^{(i, j, k, l)}(\mathbf{b})=$ $0, \forall(i, j, k, l) \in S_{1} \cup S_{2}$ also solves $\psi^{(i, j, k, l)}(\mathbf{b})=0, \forall(i, j, k, l) \in S_{0}$. Therefore, the asymmetric part $\Psi_{\mathrm{a}}$ of Hamiltonian (9) vanishes for such b. Therefore, from Definition 1 , the system (9) with the solution $\mathbf{b}$ is a symmetric lattice.

\section{Acknowledgements}

The first author (Y.D.) was partially supported by a Grant-in-Aid for Scientific Research (C), No. 19K12003 from Japan Society for the Promotion of Science (JSPS). The authors were supported by a Grant-in-Aid for Scientific Research (C), No. 19K03654 from Japan Society for the Promotion of Science (JSPS).

\section{Appendix A. Estimating velocity $v_{D B}$ of approximated traveling DB}

The map $\mathcal{T}_{\lambda}$ with an arbitrary $\lambda$ defined by Eq.(5) represents the arbitrary space shifting and sign-inverting transformation. This map corresponds to the rotation by the angle $-m \lambda$ in each $U_{m}$ component on the complex plane. Therefore, we can estimate the distance that a DB travels in the lattice from the angle that the component $U_{m}$ rotates in the complex plane.

Figure A1 shows the trajectory of component $U_{m}$ for an approximated traveling DB on the complex plane. The trajectory can be decomposed into the fast vibration corresponding to the internal vibration and the slow rotation corresponding to the propagation of a traveling DB. From the definition of map (5), the component $U_{m}$ rotates $-2 m \pi$ during the DB propagated $N$ lattice spacing. Therefore, the rotation angle $\theta_{1}=-2 m \pi / N$ of the component $U_{m}$ corresponds to an one-lattice-space propagation of the traveling DB.

We can estimate $v_{\mathrm{DB}}$ by the following steps:

(i) Perform the numerical integration of (22) and (23) for the traveling DB with a certain small perturbation $\delta l_{0}(29)$. Transform the obtained temporal evolution $q_{n}$ into $U_{m}$.

(ii) Find the time $t_{1}$ and $t_{3}$ of the first and third extreama of $\left|U_{m}\right|$.

(iii) Estimate the rotation angle $|\Delta \theta|$ between $t_{1}$ and $t_{3}$. This corresponds to the rotation angle one internal vibration:

$$
|\Delta \theta|=\arg U_{m}\left(t_{3}\right)-\arg U_{m}\left(t_{1}\right)
$$

where arg indicates the argument of complex numbers.

(iv) Calculate the velocity $v_{\mathrm{DB}}$ by

$$
v_{\mathrm{DB}}=\left|\frac{\Delta \theta}{\theta_{1}}\right|=\left|\frac{N \Delta \theta}{2 m \pi}\right| .
$$




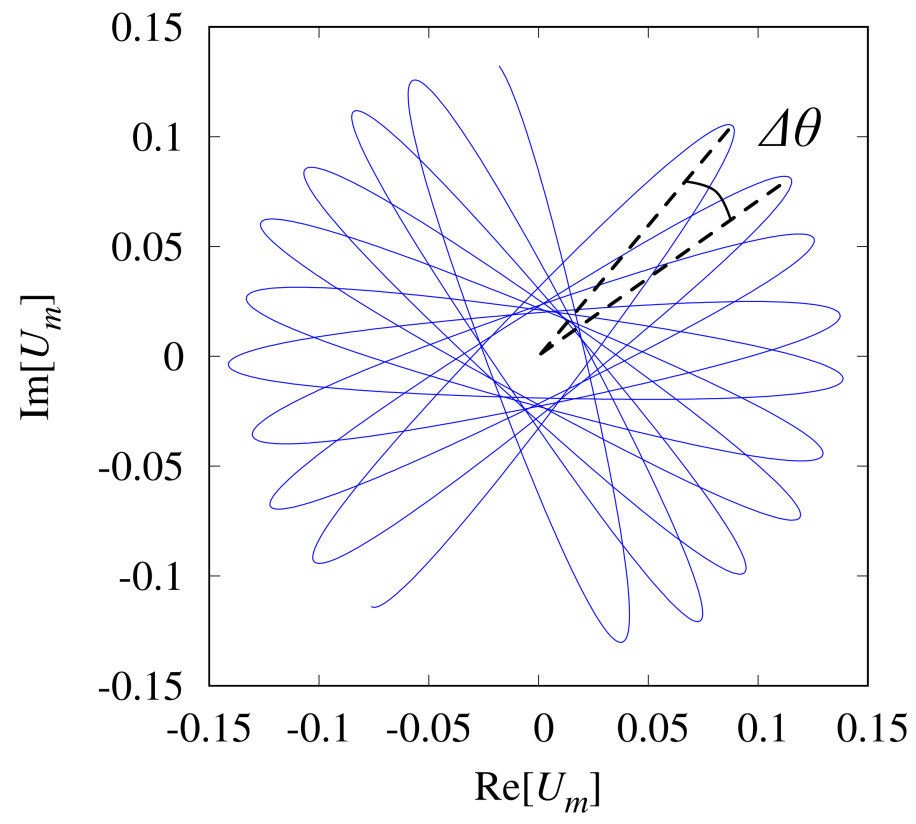

Figure A1. Trajectory of the component $U_{m}$ in the complex coordinate. $\Delta \theta$ indicates the change of angle in one internal vibration. Numerical results are the cases that $T_{\mathrm{DB}}=2, \delta l=0.02$, and $m=20$.

As is shown in Fig. 1, the velocity $v_{\mathrm{DB}}$ of approximated traveling DB is precisely proportional to $\delta l$. Therefore, we obtain the relation $v_{\mathrm{DB}}=K \delta l$. The coefficient $K$ can be calculated from one pair of $\delta l_{0}$ and $v_{\mathrm{DB}}$ for a certain $T_{\mathrm{DB}}$ following the above procedure. Finally, the $\delta l$ for desired $v_{\mathrm{DB}}$ can be obtained by $\delta l=v_{\mathrm{DB}} / K$.

\section{Appendix B. Proof of regularity of $A$}

$A$ is the $N / 4 \times N / 4$ matrix whose element is given by $A_{p q}=\cos \frac{2 \pi}{N}(p-1)(2 q-1)$. Consider the $N / 4 \times N / 4$ matrix $\bar{A}$ as follows,

$$
\bar{A}=\frac{8}{N}\left[\begin{array}{ccccc}
\frac{1}{2} A_{11} & A_{21} & A_{31} & \cdots & A_{\frac{N}{4} 1} \\
\frac{1}{2} A_{12} & A_{22} & A_{32} & \cdots & A_{\frac{N}{4}} \\
\frac{1}{2} A_{13} & A_{23} & A_{33} & \cdots & A_{\frac{N}{4} 3} \\
\vdots & \vdots & \vdots & \ddots & \vdots \\
\frac{1}{2} A_{1 \frac{N}{4}} & A_{2 \frac{N}{4}} & A_{3 \frac{N}{4}} & \cdots & A_{\frac{N}{4} \frac{N}{4}}
\end{array}\right]
$$

It can be easily shown that $A \bar{A}=\bar{A} A=I$. Therefore, $A^{-1}=\bar{A}$ and this means that $A$ is regular. 


\section{Appendix C. Proof of regularity of $P_{4} D$}

$P_{4}$ is the $N / 4 \times N / 4$ matrix given in (87) and $D$ is the $N / 4 \times N / 4$ matrix that element is given by $D_{p q}=\cos \frac{4(p-1) q \pi}{N}$. Consider the $N / 4 \times N / 4$ matrix $\bar{D}$ as follows:

$$
\bar{D}=\frac{8}{N}\left[\begin{array}{ccccc}
\frac{1}{2} D_{11} & D_{21} & D_{31} & \cdots & D_{N / 4,1} \\
\frac{1}{2} D_{12} & D_{22} & D_{32} & \cdots & D_{N / 4,2} \\
\frac{1}{2} D_{13} & D_{23} & D_{33} & \cdots & D_{N / 4,3} \\
\vdots & \vdots & \vdots & \ddots & \vdots \\
\frac{1}{2} D_{1, N / 4-1} & D_{2, N / 4-1} & D_{3, N / 4-1} & \cdots & D_{N / 4, N / 4-1} \\
\frac{1}{4} D_{1, N / 4} & \frac{1}{2} D_{2, N / 4} & \frac{1}{2} D_{3, N / 4} & \cdots & \frac{1}{2} D_{N / 4, N / 4}
\end{array}\right] .
$$

It can be shown that $\bar{D} P_{4} D=P_{4} D \bar{D}=I$. Therefore, $\left(P_{4} D\right)^{-1}=\bar{D}$ and this means that $P_{4} D$ is regular.

\section{Appendix D. Derivation of explicit solution}

At first, we consider Eq. (53). Applying the matrix $P_{1}$ from left side, we obtain

$$
P_{1} M_{1} A \mathbf{b}_{\text {odd }}=0
$$

The matrix $P_{1} M_{1}$ is given in (57). We introduce the $N / 4$-vector $\mathbf{B}$ as follows:

$$
\mathbf{B}=\left[B_{1}, B_{2}, \cdots, B_{N / 4}\right]^{T}=A \mathbf{b}_{\text {odd }}
$$

The equation (D.1) is rewritten to

$$
P_{1} M_{1} \mathbf{B}=0,
$$

and this equation leads to the relation

$$
B_{n}=-f_{N / 4}(n-1) B_{1} . \quad(n=2,3, \cdots, N / 4)
$$

Using (D.4), B can be rewritten to

$$
\mathbf{B}=B_{1}\left[1,-f_{N / 4}(1),-f_{N / 4}(2), \cdots,-f_{N / 4}(N / 4-1)\right]^{T} .
$$

Using (D.1) and (D.5), and considering Appendix C, we obtain

$$
\begin{aligned}
\mathbf{b}_{\text {odd }} & =B_{1} A^{-1}\left[1,-f_{N / 4}(1),-f_{N / 4}(2), \cdots,-f_{N / 4}(N / 4-1)\right]^{T} \\
& =B_{1} \bar{A}\left[1,-f_{N / 4}(1),-f_{N / 4}(2), \cdots,-f_{N / 4}(N / 4-1)\right]^{T} \\
& =\frac{8 B_{1}}{N}\left[\begin{array}{ccccc}
\frac{1}{2} A_{11} & A_{21} & A_{31} & \cdots & A_{\frac{N}{4} 1} \\
\frac{1}{2} A_{12} & A_{22} & A_{32} & \cdots & A_{\frac{N}{4} 2} \\
\frac{1}{2} A_{13} & A_{23} & A_{33} & \cdots & A_{\frac{N}{4} 3} \\
\vdots & \vdots & \vdots & \ddots & \vdots \\
\frac{1}{2} A_{1 \frac{N}{4}} & A_{2 \frac{N}{4}} & A_{3 \frac{N}{4}} & \cdots & A_{\frac{N}{4} \frac{N}{4}}
\end{array}\right]\left[\begin{array}{c}
1 \\
-f_{N / 4}(1) \\
-f_{N / 4}(2) \\
\vdots \\
-f_{N / 4}(N / 4-1)
\end{array}\right] .
\end{aligned}
$$


Therefore, we obtain

$$
\begin{aligned}
b_{2 s-1} & =\frac{8 B_{1}}{N}\left[\frac{1}{2} A_{1 s}-\sum_{k=1}^{N / 4-1} A_{k+1, s} f_{N / 4}(k)\right] \\
& =\frac{8 B_{1}}{N}\left[\frac{1}{2}-\sum_{k=1}^{N / 4-1} \cos \frac{2 \pi k(2 s-1)}{N} f_{N / 4}(k)\right] \\
& =\frac{8 B_{1}}{N^{2}} \frac{1}{\sin ^{2}((2 s-1) \pi / N)} . \quad(s=1,2, \cdots, N / 4)
\end{aligned}
$$

Moreover, we obtain

$$
w_{2 s-1}=\frac{b_{2 s-1}}{b_{1}}=\frac{\sin ^{2}(\pi / N)}{\sin ^{2}((2 s-1) \pi / N)} \quad(s=1,2, \cdots, N / 4) .
$$

Following additional calculations are performed for the further discussion. From the first row in Eq. (D.2), the following relation holds:

$$
B_{1}=\sum_{s^{\prime}=1}^{N / 4} b_{2 s^{\prime}-1}=b_{1} \sum_{s^{\prime}=1}^{N / 4} w_{2 s^{\prime}-1}=b_{1} N W
$$

In the last equality, we use Eq. (67). It is also obtained from Eq. (D.7) by setting $s=1$.

$$
B_{1}=\frac{b_{1}}{8} N^{2} \sin ^{2} \frac{\pi}{N}
$$

Comparing Eq.(D.9) and (D.10), we obtain

$$
W=\frac{N}{8} \sin ^{2} \frac{\pi}{N}
$$

Next, we consider Eq. (88). Applying $Q_{4} Q_{3} Q_{2} P_{2}$ from the left side, we obtain Eq. (100). We introduce $N / 4$-vector:

$$
\mathbf{G}=\left[G_{1}, G_{2}, \cdots, G_{N / 4}\right]^{T}=D \mathbf{b}_{\text {even }} .
$$

Equation (100) is rewritten to

$$
\frac{1}{8}\left[\begin{array}{cccccccc}
3 & -4 & 1 & 0 & 0 & 0 & \cdots & 0 \\
h_{N}(3) & 0 & 0 & 1 & 0 & 0 & \cdots & 0 \\
h_{N}(4) & 0 & 0 & 0 & 1 & 0 & \cdots & 0 \\
\vdots & \vdots & \vdots & \vdots & \vdots & & \ddots & \vdots \\
h_{N}(N / 4-1) & 0 & 0 & 0 & 0 & \cdots & 0 & 1 \\
h_{N}(1) & 1 & 0 & 0 & 0 & 0 & \cdots & 0 \\
h_{N}(2) & 0 & 1 & 0 & 0 & 0 & \cdots & 0
\end{array}\right] \mathbf{G}=\mathbf{g}
$$

and we obtain the relations

$$
\begin{aligned}
& 3 G_{1}-4 G_{2}+G_{3}=8 W b_{1}, \\
& G_{n}=-h_{N}(n-1) G_{1} \quad(n=2, \cdots, N / 4) .
\end{aligned}
$$

Substituting (D.15) into (D.14), we obtain

$$
G_{1}=\frac{N^{2}-4}{3 N} W b_{1}=\frac{N^{2}-4}{24} b_{1} \sin ^{2} \frac{\pi}{N},
$$


where we use Eq. (D.11) for the last equality.

Applying $P_{4}$ to (D.12) from the left side and substituting (D.15), we obtain

$$
P_{4} D \mathbf{b}_{\text {even }}=G_{1} P_{4}\left[1,-h_{N}(1),-h_{N}(2), \cdots,-h_{N}(N / 4-1)\right]^{T} .
$$

Let $P_{4}\left[1,-h_{N}(1),-h_{N}(2), \cdots,-h_{N}(N / 4-1)\right]^{T}=\left[H_{1}, H_{2}, \cdots, H_{N / 4}\right]^{T}=\mathbf{H}$. Elements of $\mathbf{H}$ are given by

$$
\begin{aligned}
& H_{1}=2\left(1-\sum_{n=1}^{N / 4-1} h_{N}(n)\right)=\frac{3 N^{2}}{2\left(N^{2}-4\right)}, \\
& H_{m}=H_{1}-1-h_{N}(m-1)=\frac{3(N-4 m+4)^{2}}{2\left(N^{2}-4\right)} \quad(m=2,3, \cdots, N / 4)
\end{aligned}
$$

Using the relation $\left(P_{4} D\right)^{-1}=\bar{D}$ (see Appendix C), we obtain

$$
\begin{aligned}
& \mathbf{b}_{\text {even }}=G_{1} \bar{D} \mathbf{H} \\
& =\frac{8 G_{1}}{N}\left[\begin{array}{ccccc}
\frac{1}{2} D_{11} & D_{21} & D_{31} & \cdots & D_{N / 4,1} \\
\frac{1}{2} D_{12} & D_{22} & D_{32} & \cdots & D_{N / 4,2} \\
\frac{1}{2} D_{13} & D_{23} & D_{33} & \cdots & D_{N / 4,3} \\
\vdots & \vdots & \vdots & \ddots & \vdots \\
\frac{1}{2} D_{1, N / 4-1} & D_{2, N / 4-1} & D_{3, N / 4-1} & \cdots & D_{N / 4, N / 4-1} \\
\frac{1}{4} D_{1, N / 4} & \frac{1}{2} D_{2, N / 4} & \frac{1}{2} D_{3, N / 4} & \cdots & \frac{1}{2} D_{N / 4, N / 4}
\end{array}\right]\left[\begin{array}{c}
H_{1} \\
H_{2} \\
\vdots \\
H_{N / 4}
\end{array}\right] .
\end{aligned}
$$

Therefore, we obtain

$$
\begin{aligned}
b_{2 s} & =\frac{8 G_{1}}{N}\left[\frac{1}{2} D_{1 s} H_{1}+\sum_{k=2}^{N / 4} D_{k s} H_{k}\right] \\
& =\frac{8 G_{1}}{N}\left[\frac{1}{2} H_{1}+\sum_{k=2}^{N / 4} \cos \frac{4(k-1) s \pi}{N} H_{k}\right] \\
& =\frac{24 G_{1}}{\left(N^{2}-4\right) \sin ^{2}(2 s \pi / N)} \quad(s=1,2, \cdots, N / 4-1) \\
b_{N / 2} & =\frac{12 G_{1}}{N^{2}-4}
\end{aligned}
$$

From the relation (D.16), we obtain

$$
\begin{aligned}
& w_{2 s}=\frac{b_{2 s}}{b_{1}}=\frac{\sin ^{2}(\pi / N)}{\sin ^{2}(2 s \pi / N)} \quad(s=1,2, \cdots, N / 4-1) \\
& w_{N / 2}=\frac{b_{N / 2}}{b_{1}}=\frac{\sin ^{2}(\pi / N)}{2} \quad(s=N / 4)
\end{aligned}
$$

Combining (D.8) and (D.22), the explicit solution is

$$
\begin{aligned}
& b_{r}=b_{1} \frac{\sin ^{2}(\pi / N)}{\sin ^{2}(r \pi / N)} \quad(r=1,2, \cdots, N / 2-1) \\
& b_{N / 2}=\frac{b_{1}}{2} \sin ^{2}(\pi / N) \quad(r=N / 2) .
\end{aligned}
$$


[1] A. J. Sievers and S. Takeno, Phys Rev Lett 61, 970 (1988).

[2] S. Flach and A. V. Gorbach, Phys. Rep. 467, 1 (2008).

[3] K. Yoshimura, Y. Doi, and M. Kimura, in Progress in Nanophotonics 3, edited by M. Ohtsu and T. Yatsui (Springer International Publishing, Cham, 2015), pp. 119-166.

[4] V. M. Burlakov, S. A. Kiselev, and V. N. Pyrkov, Phys. Rev. B 42, 4921 (1990).

[5] S. Takeno and K. Hori, J. Phys. Soc. Jpn. 60, 947 (1991).

[6] K. Hori and S. Takeno, J. Phys. Soc. Jpn. 61, 4263 (1992).

[7] K. W. Sandusky, J. B. Page, and K. E. Schmidt, Phys. Rev. B 46, 6161 (1992).

[8] S. Aubry and T. Cretegny, Phys. Nonlinear Phenom. 119, 34 (1998).

[9] S. Aubry, Phys. Nonlinear Phenom. 216, 1 (2006).

[10] K. Yoshimura and Y. Doi, Wave Motion 45, 83 (2007).

[11] J. Gómez-Gardeñes, L. M. Floría, M. Peyrard, and A. R. Bishop, Chaos 14, 1130 (2004).

[12] Y. Doi and K. Yoshimura, Phys. Rev. Lett. 117, 014101 (2016).

[13] B.-F. Feng, Y. Doi, and T. Kawahara, J. Phys. Soc. Jpn. 73, 2100 (2004).

[14] K. Yoshimura and Y. Doi, RIMS Kôkyûroku 1430, 187 (2005) (in Japanese).

[15] Y. Doi and K. Yoshimura, J. Phys. Soc. Jpn. 78, 034401 (2009).

[16] D. Bagchi, Phys Rev E 95, 032102 (2017).

[17] K. Yoshimura and Y. Doi, IEICE Tech Rep 118, 15 (2019).

[18] K. Yoshimura and Y. Doi, Proc. of the 2019 International Symposium on Nonlinear Theory and Its Applications (NOLTA2019), 399 (2019). 